\title{
Practical Absorbing Boundary Conditions for Wave Propagation on Arbitrary Domain
}

\author{
Fengru Wang ${ }^{1}$, Jerry Zhijian Yang ${ }^{1,2, *}$ and Cheng Yuan ${ }^{1}$ \\ 1 School of Mathematics and Statistics, Wuhan University, Wuhan, Hubei 430072, \\ China \\ ${ }^{2}$ Computational Science Hubei Key Laboratory, Wuhan University, Wuhan, Hubei 430072, \\ China
}

Received 9 October 2019; Accepted (in revised version) 16 March 2020

\begin{abstract}
This paper presents an absorbing boundary conditions (ABCs) for wave propagations on arbitrary computational domains. The purpose of ABCs is to eliminate the unwanted spurious reflection at the artificial boundaries and minimize the finite size effect. Traditional methods are usually complicate in theoretical derivation and implementation and work only for very limited types of boundary geometry. In contrast to other existing methods, our emphasis is placed on the ease of implementation. In particular, we propose a method for which the implementation can be done by fitting or learning from the simulation data in a larger domain, and it is insensitive to the geometry and space dimension of the computational domain. Furthermore, a stability criterion is imposed to ensure the stability of the proposed ABC. Numerical results are presented to demonstrate the effectiveness of our method.
\end{abstract}

AMS subject classifications: 60J05, 62J07, 35L05, 35L20

Key words: Absorbing boundary condition, wave equations, stability conditions.

\section{Introduction}

Many physical problems involving wave equations require solutions of essentially infinite domain in only a small region, like seismic wave propagation [11], Maxwell equation [23], Poison-Boltzmann equation [18], or inverse problem for the Helmholtz equation [1]. To solve these problems, there are generally two popular tools to overcome the difficulty of the unboundedness of the spacial domain $[2,6]$. One is the perfectly matched layer (PML) and the other is the artificial boundary method (ABM). The PML, raised by Berenger in 1994 in [4], damps the incident wave in each layer and the performance of

*Corresponding author.

Emails: wangfr@whu.edu.cn (F. R. Wang), zjyang.math@whu.edu.cn (J. Z. J. Yang), yuancheng@whu.edu.cn (C. Yuan) 
the absorbing is related to the damping rate and the number of layers [7], and is widely used in engineering like electromagnetic scattering. The main process of ABM is the design of $A B C s$, which reformulate the unbounded problem into an exact or approximate truncated domain problem. As for building ABCs of wave equations, usually the theoretical analysis plays a major part, like Engquist and Majda approximated the ABCs with a Taylor expansion in [9], and Renaut and Petersen used Chebychev-Padé approximation instead in [25]. Higdon worked directly with the difference scheme in [15,16], and Shojaei and Mossaiby extended it to finite element method [31]. Comparing to timedomain methods, spectral method also has many advantages like high accuracy and rapid convergence. And $\mathrm{ABC}$ concerning frequency domain have also been researched thoroughly [30,36]. A recent development on this region is the double absorbing boundary [12], which combines the simple formulation of PML and the high-order accuracy of artificial boundary together [24]. All these methods accord with the analytical condition that perfectly absorbs the incident wave at some certain angles of incidence.

However it should be pointed out that those methods have some imperfections in common. In the first place, it is costly in analysis, especially when we face an irregular boundary, like a disk or a polygon region, or when we need a higher-order scheme. It is always not a straightforward replication to popularize the methods, no matter restating the $\mathrm{ABC}$ in the new reference axis and approximating it by the finite difference, nor the analysis of its well-posedness. Next, in some cases it seems to be impossible to copy the boundary condition mechanically, like the corners of the rectangle, on account of their bad geometrical symmetry. Hence we have to enlarge the computation region to a more regular one and drop the results in the added region (so-called fictitious domain method). While effective, it may be too computationally expensive. At last, due to that it has been proved that a practical perfectly absorbing boundary condition can never be realized, see, e.g., [13], all these methods aim at approximating and reducing the reflection. Therefore they are effective at some angles of incidence while others not so good. In some situations, we know some information about the incidence beforehand and it limits the absorbing ability not using it. These methods exported from general analysis obviously suffer this problem. In a word, all these methods mentioned above are not problem aimed.

For these reasons, the main purpose of this paper is to give an easy-realizable method of building $\mathrm{ABC}$ for wave equations for any region, which we call learning boundary condition (LBC) in this paper, since differing from what we referred above, we obtain our results by machine learning. With the abundant research on the $\mathrm{ABC}$ based on functional approximation, the work on this perspective remains open. Our method is easily realizable, can be applied on various shapes of region, and doesn't involve the theoretical analysis of the behavior of the solution to model problems on the boundary, so it can be readily generalized to other PDEs.

The rest of this paper is organized as follows. In Section 2 we educe our method's mathematical formulation. In Section 3, we yield the learning boundary condition for the computational interval $[0,1]$, which equivalents to the exact ABCs given in [9]. In Section 
4, the method is furthered in two-dimensions of both the corner case and the boundary case. However, when applied to two-dimensions, our method results in the divergency of the solution. In Section 4.3, we fulfill our method with taking a stability condition into consideration, which greatly enhances the performance of the LBC. In Section 5, we discuss about the pathosis of the learning model, and process ridge regression to strengthen the generalization ability. Instead of adding stability condition, with this standard machine learning method the stability is gained once again independent of Section 4 . We end the paper with a conclusion in Section 6.

\section{The mathematical formulation}

Let's consider a wave propagating in $R^{2}$ :

$$
\left\{\begin{array}{l}
w_{t t}=\Delta w \\
\left.w\right|_{t=0}=f(x, y), \\
\left.w_{t}\right|_{t=0}=g(x, y),
\end{array}\right.
$$

for $(x, y) \in R^{2}$ and $t \in(0,+\infty)$ where $f$ and $g$ are both compactly supported in the left semi-plane $\{x<0\}$. Now to build an ABC for the left semi-plane, we apply Fourier transformation on $w$ to achieve:

$$
\xi^{2} \hat{w}\left(\xi, \eta_{x}, \eta_{y}\right)=\left(\eta_{x}^{2}+\eta_{y}^{2}\right) \hat{w}\left(\xi, \eta_{x}, \eta_{y}\right)
$$

where

$$
w=\int \hat{w}\left(\xi, \eta_{x}, \eta_{y}\right) \exp \left[-i\left(\xi t+\eta_{x} x+\eta_{y} y\right)\right] .
$$

But for boundary $\{(0, y)\}$, since it is an $\mathrm{ABC}$, only those waves propagating towards right go through it, therefore on the boundary we must have $\frac{\eta_{x}}{\tilde{\xi}}<0$. Then by this positivity formula (2.2) can be:

$$
\frac{\eta_{x}}{\xi} \hat{w}=-\sqrt{1-\frac{\eta_{y}^{2}}{\xi^{2}}} \hat{w},
$$

or to say

$$
\eta_{x} \hat{w}=-\operatorname{sgn}(\xi) \sqrt{\xi^{2}-\eta_{y}^{2}} \hat{w} .
$$

With inverse Fourier transformation, the operator on this boundary can be obtained:

$$
\frac{\partial w}{\partial x}=\int i \operatorname{sgn}(\xi) \sqrt{\xi^{2}-\eta_{y}^{2}} \hat{w} \exp \left[-i\left(\xi t+\eta_{y} y\right)\right] .
$$

Operator (2.6) is a non-local operator which is not applicable in computation. A practical method used widely is to approximate the operator with local operator, or to say 
differential operator. The local operator under Fourier transformation has the form of polynomials:

$$
\frac{\partial w}{\partial x}=\int i \operatorname{sgn}(\xi) \sqrt{\xi^{2}-\eta_{y}^{2}} \hat{w} \exp \left[-i\left(\xi t+\eta_{y} y\right)\right] \approx \int p\left(\xi, \eta_{y}\right) \hat{w} \exp \left[-i\left(\xi t+\eta_{y} y\right)\right],
$$

where $p \in \mathbb{P}\left(C^{2}\right)$ is a polynomial on $C^{2}$. Then the operator can be implemented numerically on methods like finite difference or finite element.

Concretely speaking, for example, after uniformly partitioning space region and half time axis into space grid and discrete time step, we can label $w_{\alpha \beta}^{k}$ being $w\left(x_{\alpha \beta}, t_{k}\right)$, the value of function $w$ on node $x_{\alpha \beta}$ in the space grid at the k-th time step. Then approximate the differential operator with finite difference method, therefore the iterative formulation to update the values of the nodes inside the region is known. To design an ABC on the boundary, the main goal is to derive the format of the formulation updating the values of the boundary node $x_{0 \beta}$. If the polynomial is chosen to be $p\left(\xi, \eta_{y}\right)=i \xi$, we can calculate the $w_{0 \beta}^{k+1}$ by forward euler:

$$
\begin{aligned}
\{ & =\frac{\partial w}{\partial x}-\int(i \xi) \hat{w} \exp \left[-i\left(\xi t+\eta_{x} x+\eta_{y} y\right)\right] \\
0 & =\frac{\partial w}{\partial x}+\frac{\partial w}{\partial t} \\
0 & =\frac{w_{0 \beta}^{k}-w_{-1 \beta}^{k}}{\Delta x}+\frac{w_{0 \beta}^{k+1}-w_{0 \beta}^{k}}{\Delta t} \\
\Longrightarrow w_{0 \beta}^{k+1} & =w_{0 \beta}^{k}-\frac{\Delta t}{\Delta x}\left(w_{0 \beta}^{k}-w_{-1 \beta}^{k}\right),
\end{aligned}
$$

which is the first order $\mathrm{ABC}$ in [9].

If the polynomial $p\left(\xi, \eta_{y}\right)$ is more complex, it will obtain a more complex formula. But all the polynomial-differentiation-difference-scheme processes lead to the same format: $w_{0 \beta}^{k+1}$, is a linear combination of those values of the nodes near $x_{0 \beta}$ in several previous time steps, which are defined as the dependent set $\mathscr{D}_{0 \beta}^{k+1}=\left\{w_{i j}^{k+1-l} \mid x_{i j}\right.$ be close to $\left.x_{0 \beta}, 1 \leq l \leq m\right\}$ :

$$
\left\{\begin{aligned}
0 & =\frac{\partial w}{\partial x}-\int p\left(\xi, \eta_{y}\right) \hat{w} \exp \left[-i\left(\xi t+\eta_{y} y\right)\right] \\
0 & =\frac{\partial w}{\partial x}-\sum c_{m_{1} m_{2}} \frac{\partial^{m_{1}+m_{2}}}{(\partial t)^{m_{1}}(\partial y)^{m_{2}}} w \\
0 & =\frac{w_{0 \beta}^{k}-w_{-1 \beta}^{k}}{\Delta x}-\sum \tilde{c}_{m_{1} m_{2}} \frac{\sum \pm w_{\alpha \beta}^{k+1-j}}{\Delta t^{m_{1}} \Delta y^{m_{2}}} \\
\Longrightarrow w_{0 \beta}^{k+1} & =\sum_{\mathscr{D}_{0 \beta}^{k+1}} a_{i j}^{l} w_{i j}^{k+1-l} \\
& =\sum_{i j}\left(a_{i j}^{1} w_{i j}^{k}+a_{i j}^{2} w_{i j}^{k-1}+a_{i j}^{3} w_{i j}^{k-2}+\cdots+a_{i j}^{m} w_{i j}^{k+1-m}\right) .
\end{aligned}\right.
$$


In precious work, usually a specific polynomial is given in (2.7), then the analysis of the reflection coefficients follows. However as mentioned in Section 1, instead of theoretical analysis, we apply machine learning to obtain the formulation. Assuming there is a distribution $\rho$ of the wave vector and wave length, we choose the optimal coefficients to minimize the population risk for this model:

$$
a_{i j}^{l} w_{i j}^{k+1-l} \in \min \mathbb{E}_{\rho}\left|i \operatorname{sgn}(\xi) \sqrt{\xi^{2}-\eta_{y}^{2}} \hat{w}-p\left(\xi, \eta_{y}\right) \hat{w}\right|^{2} .
$$

By Parseval's principle the summary on frequency equals the summary on time:

$$
a_{i j}^{l} w_{i j}^{k+1-l} \in \min \mathbb{E}_{\rho}\left|w_{0 \beta}^{k+1}-\sum_{\mathscr{D}_{0 \beta}^{k+1}} b_{i j}^{l} w_{i j}^{k+1-l}\right|^{2} \triangleq \operatorname{Loss}_{p o p}\left(b_{i j}^{l}\right) .
$$

To reduce the difficulty of the computation in (2.11), we replace the population risk with the empirical risk: Solve the differential equations with several initial condition obeying the distribution $\rho$ on a region larger enough that the wave propagates through the previous boundary but does not reach the new boundary. Then let $\left\{{ }_{m} u_{\alpha \beta}^{k}\right\}$ be the solution corresponding to each initial condition. Substituting $\left\{{ }_{m} u_{\alpha \beta}^{k}\right\}$ into (2.11) leads to a least square problem:

$$
a_{i j}^{l} \in \min \sum_{m, k}\left|m u_{0 \beta}^{k+1}-\sum_{\mathscr{D}_{0 \beta}^{k+1}} b_{i j m}^{l} u_{i j}^{k+1-l}\right|^{2} \triangleq \operatorname{Loss}_{e m p}\left(b_{i j}^{l}\right),
$$

which can be easily solved by generalized inverse.

However, like what we are going to discuss about in Section 4.3 and Section 5, not always does the least square regression work. For stability or the generalization ability, some constraints must be set. Here we bring $l^{1}$ regularization and Tikhonov regularization in:

$$
\begin{array}{ll}
l^{1}: & a_{i j}^{l} \in \min _{\left\|b_{\alpha \beta}^{j}\right\|_{1}<r} \operatorname{Loss}_{e m p}\left(b_{i j}^{l}\right), \\
\text { Tikhonov: } & a_{i j}^{l} \in \min _{\left\|b_{\alpha \beta}^{j}\right\|_{2}<r} \operatorname{Loss}_{e m p}\left(b_{i j}^{l}\right) .
\end{array}
$$

The $l^{1}$ regularization is also known as "Least Absolute Shrinkage and Selection Operator" (or LASSO) problem, see [32], which is a important and hot topic in statistics and machine learning. Many useful algorithms have been given, such as the famous iterative thresholding algorithm (ISTA), first derived in [27] (see also [28]), later [29], and first placed on solid mathematical grounds in [17]. 
However, here $l^{1}$ regularization is not for the sake of the sparsity of $a_{i j}^{l}$ and neither do we need here. On the one hand, the support set of the coefficient can be appointed a prior since the dependent set is chosen artificially, therefore the unrelated data can be excluded. On the other hand, without regard to the sparsity we can use interior point method instead of those algorithms mentioned previously, which is easier to implement and more stable. Replacing the constraint $\left\|b_{\alpha \beta}^{j}\right\|_{1}<r$ with a group of linear constraint, we have:

$$
a_{i j}^{l} \in \min _{l_{q}\left(b_{i j}^{l}\right)<r} \operatorname{Loss}_{e m p}\left(b_{i j}^{l}\right),
$$

where $l_{q}(b)= \pm b_{1} \pm b_{2} \pm b_{3} \pm \cdots \pm b_{q}$ are all the linear functionals of $R^{q}$ whose coefficients are \pm 1 . Then we can set a group of logarithmic barrier functions:

$$
L\left(b_{i j}^{l} ; \mu\right)=\operatorname{Loss}_{e m p}\left(b_{i j}^{l}\right)-\sum_{q} \mu_{q} \times \ln \left(r-l_{q}\left(b_{i j}^{l}\right)\right),
$$

and then solve the unconstrained optimization of $\left(b_{i j}^{l} ; \mu\right)$ by steepest descent method. Here $\mu$ is a small positive scalar. As $\mu$ converges to zero the minimum point of $L\left(b_{i j}^{l} ; \mu\right)$ should converge to the solution of original problem. Therefore we iteratively substitute $\mu$ by $\mu \times 0.1$ and solve the new unconstrained optimization problem until stopping criteria is satisfied, then we gain a numerical solution of the former constrained optimization.

As for Tikhonov regularization, the motivation and the background will be given later in section 5 , while the solution of this problem is pretty simple: replacing the generalized inverse $\left(U^{T} U\right)^{-1} U^{T}$ of a matrix $U$ with $\left(U^{T} U+\lambda I\right)^{-1} U^{T}$, where $\lambda$ is the Lagrangian multiplier. Notice that assuming the largest and smallest singular value of $U^{T} U$ are $\sigma 1$ and $\sigma 2$, its condition number is $\sigma 1 / \sigma 2$ while $\left(U^{T} U+\lambda I\right)^{\prime} \mathrm{s}$ is $(\sigma 1+\lambda) /(\sigma 2+\lambda)$, which means less ill-conditioned.

\section{The one-dimension case}

In this section we perform a simple experiments on interval $[0,1]$ which leads to a boundary condition $L b c 1$ (Learning boundary condition). Then it will be pointed out that it is exactly the difference form of the perfect absorbing boundary condition.

Let $x_{n}=n d x, n \in\{0,1,2, \cdots, N\}, t_{k}=k d t, k \in Z$ be the uniformly spaced grid. We partition the interval $[0,1]$ into 1000 nodes and take 0.0009 as the time-step $(\Delta x=0.001, \Delta t=0.0009)$. Different schemes can be employed to approximate the wave differential operator [19]. We use the centre difference quotients:

$$
\frac{w_{n+1}^{k}+w_{n-1}^{k}-2 w_{n}^{k}}{(\Delta x)^{2}}=\frac{w_{n}^{k+1}+w_{n}^{k-1}-2 w_{n}^{k}}{(\Delta t)^{2}},
$$

which leads to the recursive formula:

$$
w_{n}^{k+1}=\left(w_{n+1}^{k}+w_{n-1}^{k}-2 w_{n}^{k}\right) \frac{(\Delta t)^{2}}{(\Delta x)^{2}}+2 w_{n}^{k}-w_{n}^{k-1}, \quad n \in\{1,2,3, \cdots, N-1\}, \quad k \in \mathbb{N} .
$$


Following the procedure given in Section 2, we have the following scheme with Lbc at $x=1$ and a fixed boundary at $x=0$ :

$$
\left\{\begin{array}{l}
w_{0}^{k+1}=0 \\
w_{n}^{k+1}=\left(w_{n+1}^{k}+w_{n-1}^{k}-2 w_{n}^{k}\right) \frac{(\Delta t)^{2}}{(\Delta x)^{2}}+2 w_{n}^{k}-w_{n}^{k-1}, \quad n \in\{1, \cdots, N-1\}, \\
w_{N}^{k+1}=\sum_{\mathscr{D}_{N}^{k+1}} a_{i}^{j} \times w_{i}^{k+1-j} .
\end{array}\right.
$$

The learning function for constructing the learning set are given as:

$$
\begin{aligned}
& f_{l}(x)= \begin{cases}\cos (5(x-0.5)), & x \in[0.4,0.6], \\
0, & \text { else, }\end{cases} \\
& g_{l}(x)= \begin{cases}\frac{\pi}{2} \sin (5(x-0.5)), & x \in[0.4,0.6], \\
0, & \text { else, }\end{cases}
\end{aligned}
$$

and the dependent set of $w_{N}^{k+1}$ is $\left\{w_{N}^{k}, w_{N-1}^{k}\right\}$. Then the dependent coefficients $a_{\alpha}^{l}$ is derived:

$$
L b c 1: \quad w_{1000}^{k+1}=0.8999538 \times w_{999}^{k}+0.1000463 \times w_{1000}^{k} .
$$

Recall that the perfectly absorbing boundary condition given in Eq. (2.6) degenerates in one-dimension case as $\eta_{y}=0$. And for those incident wave to be absorbed on $x=1$ we have $\frac{\eta_{x}}{\tilde{\xi}}<0$. So now the perfectly absorbing boundary condition on $x=1$ is:

$$
\left.\frac{\partial}{\partial x} w\right|_{x=1}+\int_{\xi}(-i \xi) \times \hat{w}(1, \xi) e^{-i \xi t} d \xi=0
$$

which is:

$$
\left.\left(\frac{\partial}{\partial x}+\frac{\partial}{\partial t}\right) w\right|_{x=1}=0
$$

writing in difference form:

$$
w_{N}^{k+1}=(1-\Delta t / \Delta x) \times w_{N-1}^{k}+\Delta t / \Delta x \times w_{N^{\prime}}^{k}, \quad w_{N}^{k+1}=0.9 \times w_{N-1}^{k}+0.1 \times w_{N}^{k},
$$

which is almost the same one of $L b c 1$. It implies the method we applied here works, since we have reached the same formulate coming from differencing the perfectly absorbing condition by only performing regression, a standard method used in machine learning, on data set, which motivates us to put it into higher dimension space.

\section{The two-dimension case}

In this section, Lbcs in $R^{2}$ are discussed. The Lbc for corner, the stability condition and $\mathrm{Lbc}$ for a general planar region are provided in turn. 


\subsection{Learning corner condition}

Usually when people construct the $\mathrm{ABC}$ for a bounded rectangle region on the plane we only take the straight boundary into consideration but rarely consider the corner. However, it is much harder to analyse the property of the equation on the neighborhood of a corner and we can just simply treat the corner nodes as boundary nodes of one of its two sides [3]. This handling leads to the corner reflection, which is verified in this section. But the machine learning method can easily overcome this difficulty: Specifically speaking, if the nodes are sequentially numbered (node $(\alpha d x, \beta d y)$ is numbered to be $x_{\alpha \beta}$ ), assume its four corner nodes is iterated analogously like the boundary nodes of one-dim interval, given by

$$
\begin{aligned}
& w_{00}^{k+1}=\sum_{\mathscr{D}_{00}^{k+1}} a_{i j}^{l} \times w_{i j}^{k+1-l}, \\
& w_{0 N}^{k+1}=\sum_{\mathscr{D}_{0 N}^{k+1}} b_{i j}^{l} \times w_{i j}^{k+1-l}, \\
& w_{N 0}^{k+1}=\sum_{\mathscr{D}_{N 0}^{k+1}} c_{i j}^{l} \times w_{i j}^{k+1-l}, \\
& w_{N N}^{k+1}=\sum_{\mathscr{D}_{N N}^{k+1}} d_{i j}^{l} \times w_{i j}^{k+1-l} .
\end{aligned}
$$

Then following the same procedure we can obtain the learning boundary condition for the corner.

\subsubsection{Example 3}

We partition a square into $1200 \times 1200$ nodes with $\Delta t=0.6 \Delta x$ as the time-step. The learning function for constructing the learning set is

$$
\begin{aligned}
& f_{l}(x, y)= \begin{cases}100 \cos [12 \pi(4 r+x)], & r=|(x, y)-(0.5,0.5)| \leq 0.25, \\
0, & \text { else, }\end{cases} \\
& g_{l}(x, y)= \begin{cases}500 \sin [12 \pi(4 r+x)], & r=|(x, y)-(0.5,0.5)| \leq 0.25, \\
0, & \text { else, }\end{cases}
\end{aligned}
$$

and the dependent set of $w_{00}^{k+1}$ is $\left\{w_{00}^{k}, w_{10}^{k}, w_{01}^{k}, w_{11}^{k}\right\}$. The dependent coefficients we acquire reads:

$$
L b c 3: \quad w_{00}^{k+1}=w_{00}^{k} \times 0.0519+w_{10}^{k} \times 0.45050+w_{01}^{k} \times-0.10347+w_{11}^{k} \times 0.60047 .
$$

Then learning boundary condition $L b c 3$ is built on the four corners of the rectangle $[0,1] \times$ $[1,0]$ symmetrically and the normal boundary nodes, those on the straight boundary, are setted to equal the precise values, calculated by solving the equation on a larger region (fictitious domain method). Thus we eliminate the influence of the other nodes and all 
the reflection is produced only by the $L b c 3$. Also the $L b c 4$ given in Example 4 (Section 4.1.2) below, the traditional handling of the corner nodes and the fix boundary condition are applied in the same way for comparison. The numerical results of these four boundary condition are illustrated in Fig. 1. Analysis of the results is provided in the end of Example 4 (Section 4.1.2).

\subsubsection{Example 4}

In this example we enlarge the dependent set of the corner node. This time $\mathscr{D}_{0,0}^{k+1}$ is: $\left\{w_{10}^{k+1}, w_{01}^{k+1}, w_{11}^{k+1}, w_{00}^{k}, w_{10}^{k}, w_{01}^{k}, w_{11}^{k}\right\}$ and we have:

$$
\begin{aligned}
L b c 4: w_{00}^{k+1}= & -0.08732 \times w_{10}^{k+1}+0.07390 \times w_{01}^{k+1}+0.03818 \times w_{11}^{k+1} \\
& +0.00196 \times w_{00}^{k}+0.60480 \times w_{10}^{k}+0.57410 \times w_{01}^{k} \\
& -0.20620 \times w_{11}^{k} .
\end{aligned}
$$

The numerical results of this boundary condition are illustrated in Fig. 1. As we can see in this figure, both $L b c 3$ and $L b c 4$ have little reflection. Contrasting with the method used before, which ignores that the reflection is produced by the two sides of the corner, the learning boundary condition for the corner nodes does reduce the reflection visibly.

\subsection{Lbc for irregular boundary}

Suppose that in region $D$

$$
\left\{\begin{array}{l}
w_{t t}=\Delta w \\
\left.w\right|_{t=0}=f(x, y) \\
\left.w_{x}\right|_{t=0}=g(x, y) .
\end{array}\right.
$$

where $D=\left\{(x, y) \mid-\infty<x<\infty, f_{d}(x) \leq y \leq f_{u}(x)\right\}$. Let $\Gamma_{1}=\left\{\left(x, f_{d}(x)\right\}\right.$ and $\Gamma_{2}=\left\{\left(x, f_{u}(x)\right\}\right.$. We now want to build the learning boundary condition for $\Gamma_{1}$ and $\Gamma_{2}$. Assume

$$
\begin{aligned}
& w_{n d(n)}^{k+1}=\sum_{\mathscr{D}_{n d(n)}^{k+1}} a_{i j, n}^{l} \times w_{i j}^{k+1-l}, \\
& w_{n u(n)}^{k+1}=\sum_{\mathscr{D}_{n u(n)}^{k+1}} b_{i j, n}^{l} \times w_{i j}^{k+1-l},
\end{aligned}
$$

where $\left\{x_{n d(n)}\right\}$ and $\left\{x_{n, u(n)}\right\}$ are two sequences of point in space grid to approximate the boundary $\Gamma_{1}$ and $\Gamma_{2}$.

We construct two different irregular boundaries in this paper: a polygonal line and a convex curve. With the space grid of $1200 \times 1200$ nodes uniformly partitioning the 

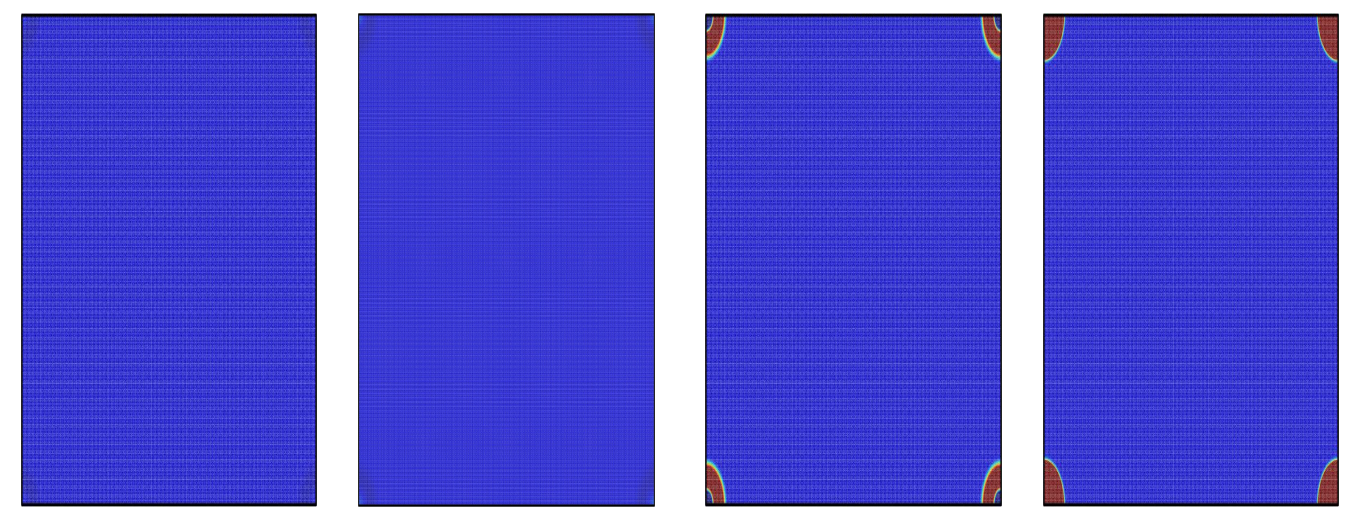

$K=640$
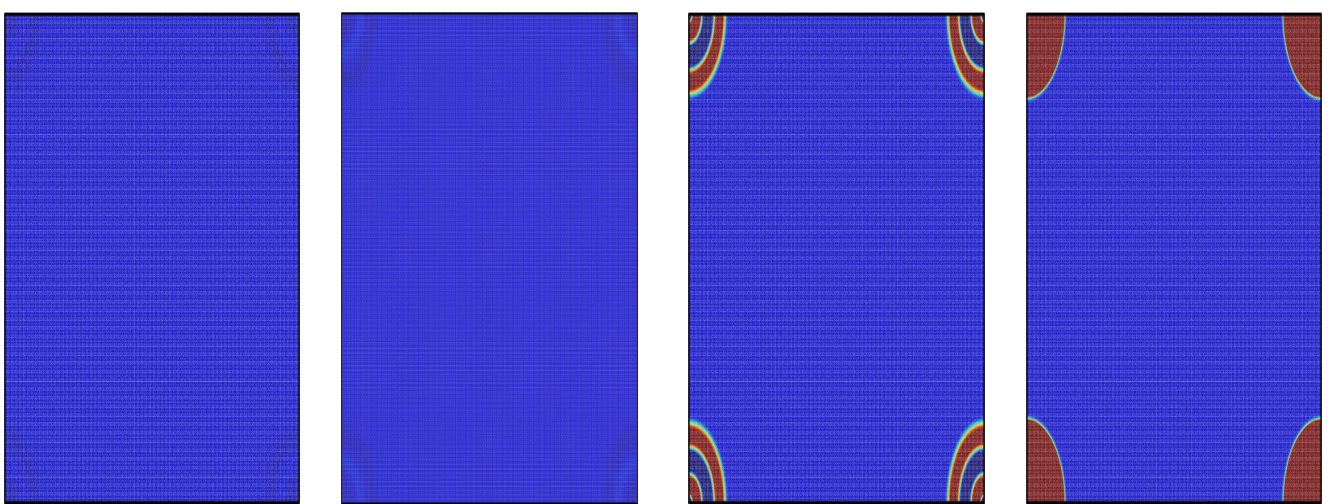

$K=720$
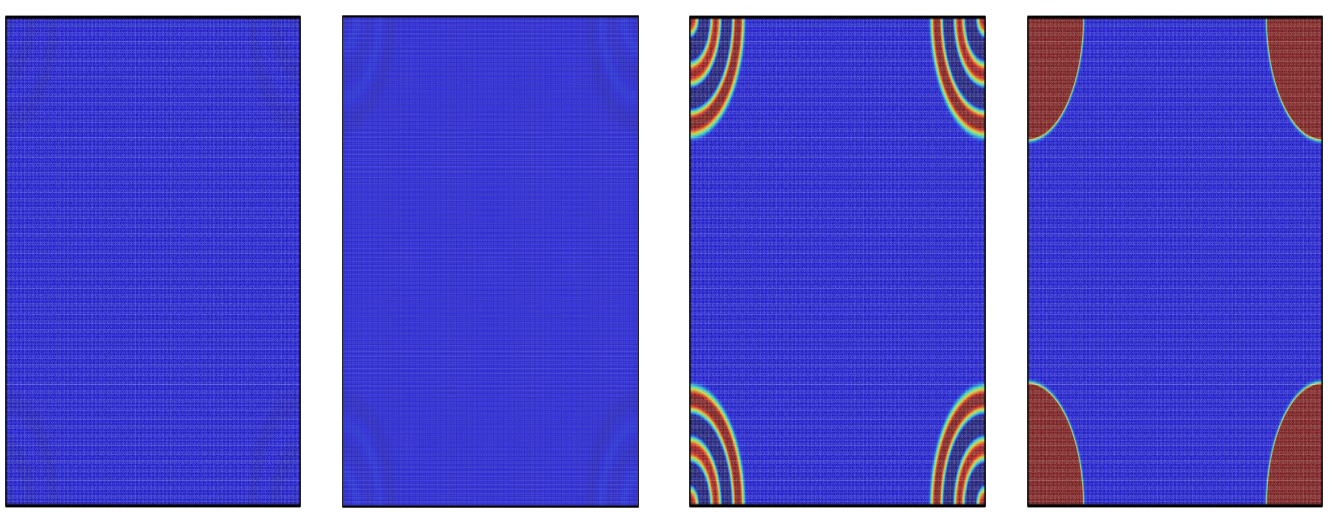

$K=800$

Figure 1: These are the result of Example 3 and Example 4. The first and the second columns correspond to the $L b c 3$ and $L b c 4$ we obtain while the third comes from treating the corner code as one of the upper and lower boundary, and the fourth is fixing $w_{00}^{k}=w_{N 0}^{k}=w_{0 N}^{k}=w_{N N}^{k}=0$. 

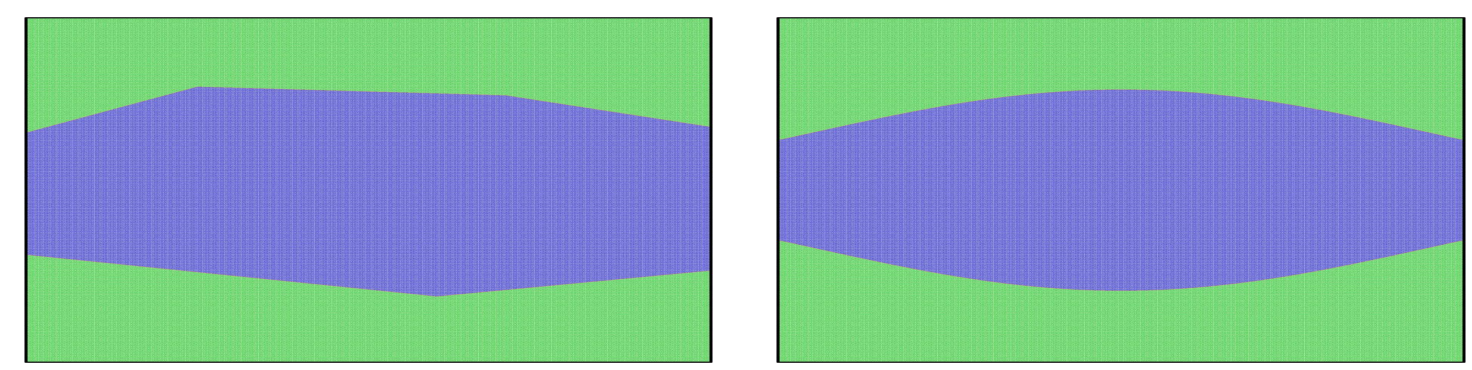

Figure 2: The figure on the right is the shape of the polygonal line boundary given in Section 4.2 and the left is the one of the curve. The blue region is the region of computation and the learning boundary condition is built on the boundary of the blue region and the green region.

rectangle $[0,1] \times[0,1]$, concrete forms of these two boundaries are:

$$
\begin{aligned}
& \text { Polygonal line: } \begin{cases}u(n)=800+i n t[16 n / 30], & 0 \leq n \leq 300, \\
u(n)=960-i n t[n / 18-50 / 3], & 300 \leq n \leq 840, \\
u(n)=930-i n t[11 n / 36-770 / 3], & 840 \leq n \leq 1200, \\
d(n)=375-i n t[29 n / 144], & 0 \leq n \leq 720, \\
d(n)=230+i n t[9 n / 48-135], & 720 \leq n \leq 1200,\end{cases} \\
& \text { Curve: } \begin{cases}u(n)=800+\operatorname{int}[\cos (\pi(n-600) / 1080) \times 150], & 0 \leq n \leq 1200, \\
d(n)=400-i n t[\cos (\pi(n-600) / 1080) \times 150], & 0 \leq n \leq 1200 .\end{cases}
\end{aligned}
$$

The shape of these two boundary is given in Fig. 2.

In the meantime all the examples have been carried out for the straight line boundary $\Gamma_{1}=\{(x, 0.25)\}$ and $\Gamma_{2}=\{(x, 0.75)\}$ too.

\subsection{The stability criterion}

In a discrete system, since we have made the linearly dependence assumption, the iterative formula can be written into a matrix form. Let $x_{p}^{k}=w_{i j}^{k}$, where $p=N(i-1)+j$. For example, without the loss of generality, we can assume that the dependent set only retrospect two time layers. As $x_{p}^{k+1}$ is linearly dependent on $x_{p}^{k}$ and $x_{p}^{k-1}$, the recursive formula together with the boundary condition can be written into matrix form as:

$$
x^{k+1}=A x^{k}+B x^{k-1} .
$$

Let $y^{k+1}=\left(x^{k+1 T}, x^{k T}\right)^{T}$, then we have

$$
y^{k+1}=C y^{k}
$$

where

$$
C=\left(\begin{array}{cc}
A^{2}+B & A B \\
A & B
\end{array}\right)
$$


Then the spectral radius of the matrix $C$ measures the stability of the iterative formulation. We have calculated the matrix $C$ for a small squared grid and evaluate its eigenvalues by power method, namely:

$$
\left\{\begin{array}{l}
y^{k}=\frac{x^{k}}{\max \left|x^{k}\right|} \\
x^{k+1}=C y^{k}
\end{array}\right.
$$

Recall the boundary condition for the left semi-plane:

$$
w_{0 \beta}^{k+1}=\sum_{\mathscr{D}_{0 \beta}^{k+1}} a_{i j}^{l} \times w_{i j}^{k+1-l} .
$$

The first constraint we make is to limit the dependent set of $w_{0 \beta}^{l}$ on those points having the same vertical coordinates $(j=\beta)$ :

$$
w_{0 \beta}^{k+1}=\sum_{i \leq 0, l \geq 1} a_{i \beta}^{l} \times w_{i \beta}^{k+1-l} .
$$

This is a reasonable assumption since the derivatives of $d y$ in boundary condition (2.7) can be transformed into derivatives of $d x$ and $d t$ by $\partial_{y}^{2}=\partial_{t}^{2}-\partial_{x}^{2}$. Then by formula (4.9) we assume that the eigenvectors of the iterative system take the form of:

$$
w_{\alpha \beta}^{k}=\lambda_{q}^{k} \int_{\eta_{y}} \hat{w}_{\alpha}\left(\eta_{y}\right) \exp \left(-i \eta_{y} \beta \Delta y\right) .
$$

We substitute (4.14) into both the centre difference scheme and the boundary condition (4.13). We have acquired that:

$$
\left\{\begin{array}{l}
\hat{w}_{\alpha+1}+\hat{w}_{\alpha-1}=\left[\frac{\Delta x^{2}\left(\lambda_{q}+\lambda_{q}^{-1}-2\right)}{\Delta t^{2}}+\frac{\Delta x^{2}\left(2-2 \cos \left(\eta_{y} \beta \Delta y\right)\right)}{\Delta y^{2}}+2\right] \hat{w}_{\alpha 1} \\
\hat{w}_{0}=\sum_{i \leq 0, l \geq 1} a_{i \beta}^{l} \times \lambda_{q}^{-l} \hat{w}_{i} .
\end{array}\right.
$$

Theorem 4.1. For boundary condition (4.13), if the following criterion is satisfied:

$$
|| a_{i \beta}^{l} \|_{1}=\sum_{i l}\left|a_{i \beta}^{l}\right|<1
$$

and the initial value in the Cauchy problem (2.1) has only the wave propagating towards right and a compact supported set, then the boundary condition together with centre difference scheme is a stable numerical scheme for wave equations in the left semi-plane. 
This criterion has been used to prove the stability for some first-order hyperbolic systems, see Gustafsson, Kreiss, and Sundström [5] and Michelson [22], and been regarded as a guideline for constructing and analysing boundary condition for wave equations, see Renaut [25, 26], Higdon [14-16] and Givoli and Cohen [10] and interface condition [34]. In $[33,35]$ a exhaustive discuss is given by Trefethen concerning the stability of frog-leap algorithm. It is also closed related with the LASSO problem in sparse models, but the difference there lies that here Theorem 4.1 does not aim at the sparsity of the coefficients $a_{i \beta}^{l}$. Inequality (4.16) describes the boundary between the scheme being stable in time and those not, and usually the solution sneaks over this boundary when we replace the population risk of the problem $\operatorname{Loss}_{p o p}\left(b_{i j}^{l}\right)$ with the empirical risk $\operatorname{Loss}_{e m p}\left(b_{i j}^{l}\right)$, therefore an extra constraint needs built. since the fourier transformation is used in form (4.14), the proof can not be generalized to irregular boundary. But as we will see in Section 4.4.4 and Section 4.4.5, it does solve the stability of this numerical algorithm.

Proof. To proof the stability in time for the boundary condition is to proof that all eigenvalues of the iterative system have module less than 1 , by formula (4.9). since the matrix in formula (4.9) is not a symmetric matrix, the eigenvalue whose module equals 1 has to be specially treated. Let's assume that there is an eigenvalue $\lambda_{1}$ whose module is greater than or equal to 1 , then by reductio ad absurdum we will proof there are no non-trivial solution of $\hat{w}_{\alpha}$.

Take absolute value in both side of the second formula in (4.15), since $\left|\lambda_{1}\right| \geq 1$, by cauchy inequality:

$$
\begin{aligned}
\left|\hat{w}_{0}\right| & =\left|\sum_{i \leq 0, l \geq 1} a_{i \beta}^{l} \times \lambda_{q}^{-l} \hat{w}_{i}\right| \leq\left\|a_{i \beta}^{l}\right\|_{1} \times\left\|\lambda_{1}^{-l} \hat{w}_{i}\right\|_{\infty} \\
& <1 \times\left\|\hat{w}_{i}\right\|_{\infty}<\max _{\alpha}\left|\hat{w}_{\alpha}\right|,
\end{aligned}
$$

which means that $\left|\hat{w}_{0}\right|$ is not the maximum. But since the wave propagates towards right and is compactly supported, there must be a maximum in $\left|\hat{w}_{\alpha}\right|$ for $\alpha<0$. Let $\left|\hat{w}_{\gamma}\right|$ be the maximum, then take the absolute value in both side of the first formula in (4.15) with $\alpha=\gamma$ for $\gamma<0$ :

$$
\begin{aligned}
& 2\left|\hat{w}_{\gamma}\right| \geq\left|\hat{w}_{\gamma+1}+\hat{w}_{\gamma-1}\right| \\
= & \left|\left[\left(\frac{\Delta x}{\Delta t}\right)^{2}\left(\lambda_{1}+\lambda_{1}^{-1}-2\right)+\left(\frac{\Delta x}{\Delta y}\right)^{2}\left(2-2 \cos \left(\eta_{y} \beta \Delta y\right)\right)+2\right] \hat{w}_{\gamma}\right| \\
\geq & \mathbb{R}\left[\left(\frac{\Delta x}{\Delta t}\right)^{2}\left(\lambda_{1}+\lambda_{1}^{-1}-2\right)+\left(\frac{\Delta x}{\Delta y}\right)^{2}\left(2-2 \cos \left(\eta_{y} \beta \Delta y\right)\right)+2\right]\left|\hat{w}_{\gamma}\right| .
\end{aligned}
$$

Therefore both $\mathbb{R}\left(\lambda_{q}+\lambda_{q}^{-1}-2\right)$ and $\mathbb{R}\left(2-2 \cos \left(\eta_{y} \beta \Delta y\right)\right)$ are zero, which means $\lambda_{1}=1$ and $\eta_{y}=0$. Apparently that is a constant solution for the wave equations and is stable in time trivially. 


\subsection{Numerical experiments}

\subsubsection{Example 5}

In this experiment, with the space grid given in Section 4.2 , the ratio of the time step and the space step is 0.6 , and the learning function is

$$
\left\{\begin{array}{l}
f_{l}(x, y)=250\{0.1 \cos [60 \pi(x+5 y)]+0.1 \cos [60 \pi(x+y / 5)]+\cos [60 \pi r]\}, \\
g_{l}(x, y)=250\{0.1 \cos [60 \pi(x+2 y)]+0.1 \cos [60 \pi(x-2 y)]+\cos [60 \pi r]\},
\end{array}\right.
$$

supported in $r<0.125$ where $r=\left|(x, y)-\left(\frac{1}{2}, \frac{1}{2}\right)\right|_{2}$.

Let $\mathscr{D}_{n d(n)}^{k+1}$ be $\left\{w_{n d(n)}^{k}, w_{n d(n)+1}^{k}, w_{n d(n)+2}^{k}, w_{n d(n)}^{k-1}, w_{n d(n)+1}^{k-1}, w_{n d(n)+2}^{k-1}\right\}$ and $\mathscr{D}_{n u(n)}^{k+1}$ be $\left\{w_{n u(n)}^{k}, w_{n u(n)-1}^{k}, w_{n u(n)-2}^{k}, w_{n u(n)}^{k-1}, w_{n u(n)-1}^{k-1}, w_{n u(n)-2}^{k-1}\right\}$. After obtaining the dependent coefficients $\left\{a_{i j, n}^{l}\right\}$ and $\left\{b_{i j, n}^{l}\right\}$ without the stability condition (4.16), we test our learning boundary condition by solving the initial value problem:

$$
\begin{cases}w_{t t}=w_{x x}+w_{y y}, & \\ \left.w\right|_{t=0}=2000 \times \cos (120 \pi r), & r<0.15 \\ \left.w_{x}\right|_{t=0}=1000 \times \cos (120 \pi r), & r<0.15\end{cases}
$$

The numerical results are illustrated in Figs. 3, 4 and 5. As we can see, the boundary condition is unstable. Even in the case where we treat with straight boundary, there is a distinct reflected wave produced by the learning boundary condition, which means this naive replication of the one-dim case fails without the stability condition.

\subsubsection{Example 6}

With the same space and time grid as given in Example 5, same dependent set $\mathscr{D}_{n, d(n)}^{k+1}$ and $\mathscr{D}_{n, u(n)}^{k+1}$ and the same learning function:

$$
\left\{\begin{array}{l}
f_{l}(x, y)=250\{0.1 \times \cos [60 \pi(x+5 y)]+0.1 \times \cos [60 \pi(x+y / 5)]+\cos [60 \pi r]\}, \\
g_{l}(x, y)=250\{0.1 \times \cos [60 \pi(x+2 y)]+0.1 \times \cos [60 \pi(x-2 y)]+\cos [60 \pi r]\},
\end{array}\right.
$$

supported in $r<0.125$, the same learning boundary condition as the one in Example 5 is obtained. But this time the initial value is exactly the same as the learning function:

$$
\left\{\begin{array}{l}
w_{t t}=w_{x x}+w_{y y}, \\
\left.w\right|_{t=0}=250\{0.1 \cos [60 \pi(x+5 y)]+0.1 \cos [60 \pi(x+y / 5)]+\cos [60 \pi r]\}, \\
\left.w_{x}\right|_{t=0}=250\{0.1 \cos [60 \pi(x+2 y)]+0.1 \cos [60 \pi(x-2 y)]+\cos [60 \pi r],
\end{array}\right.
$$

supported in $r<0.125$.

The numerical results are illustrated in Figs. 6, 7 and 8. The sameness of the learning data and test data eliminates the generalized error between the population risk (2.11) and the empirical risk (2.12), which lets the failure of the experiments lead us directly to the instability. 

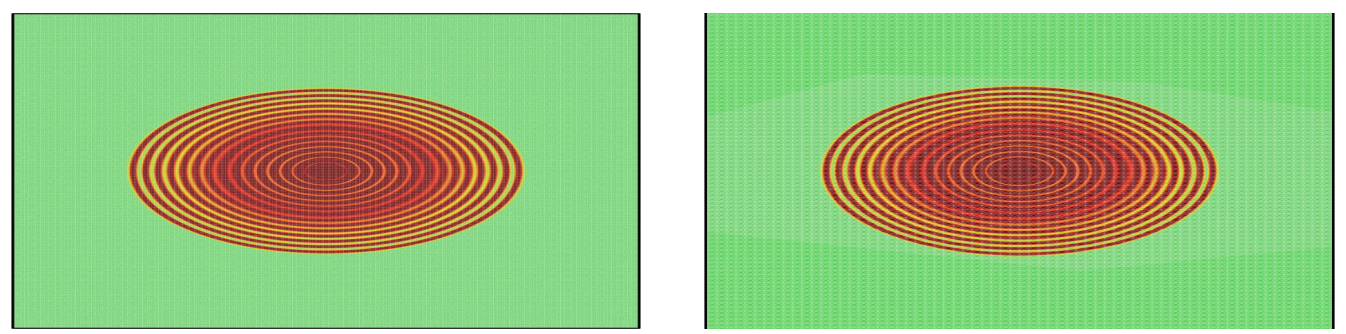

$K=280$
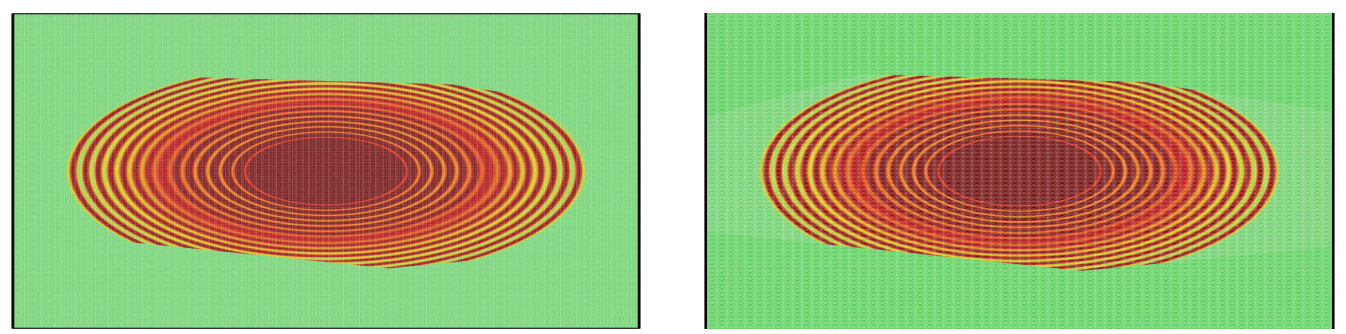

$$
K=440
$$
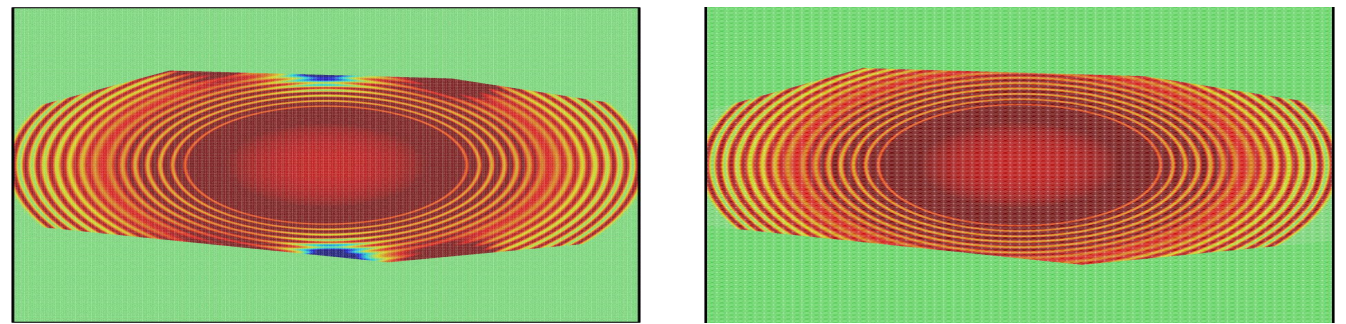

$$
K=600
$$
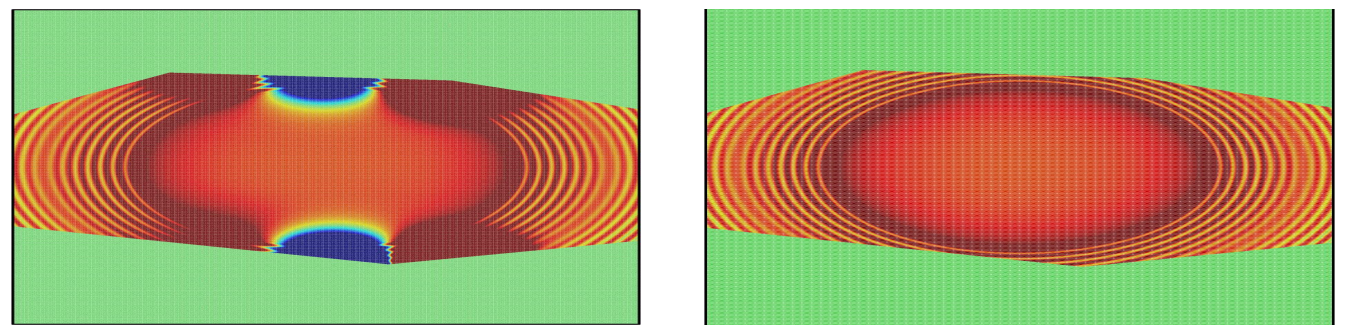

$$
K=760
$$

Figure 3: These are the results of Example 5, building learning boundary condition for the ploygonal line boundary. The first column is the numerical solution and the second column corresponds to the precise solution acquired from solving the equation in a larger region.

\subsubsection{Example 7}

We build a simple example with only 10000 nodes to deleave the rectangle $[0,1] \times[0,1]$. The ratio of the time and space step is 0.6. The learning function is:

$$
\left\{\begin{array}{l}
f_{l}(x, y)=250\{0.1 \cos [6 \pi(x+5 y)]+0.1 \cos [6 \pi(x+y / 5)]+\cos [6 \pi r]\}, \\
g_{l}(x, y)=250\{0.1 \cos [6 \pi(x+2 y)]+0.1 \cos [6 \pi(x-2 y)]+\cos [6 \pi r]\},
\end{array}\right.
$$



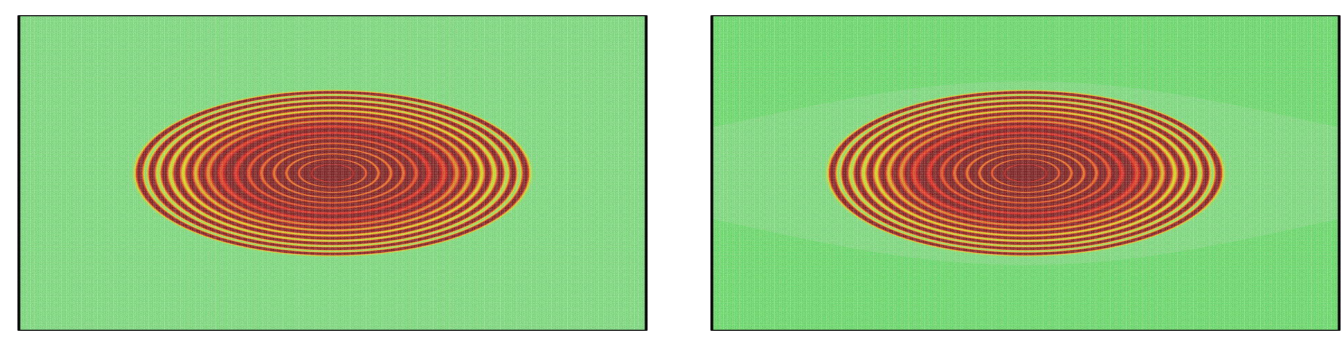

$K=280$
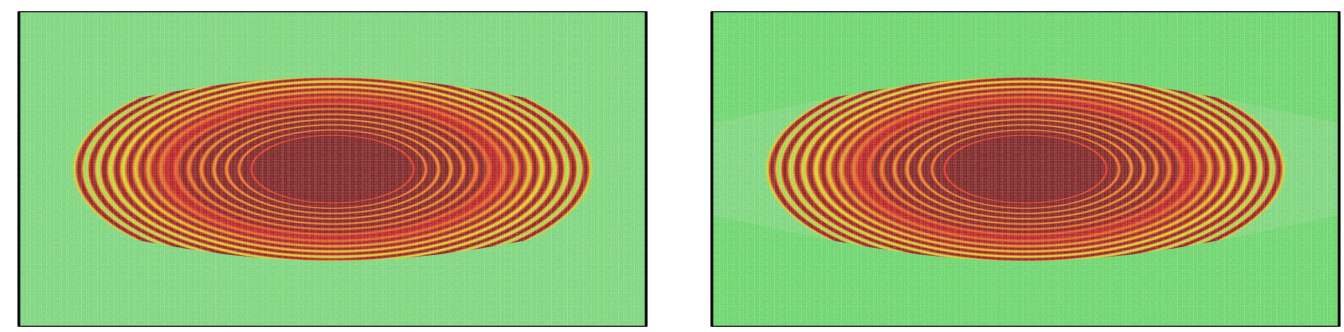

$$
K=440
$$

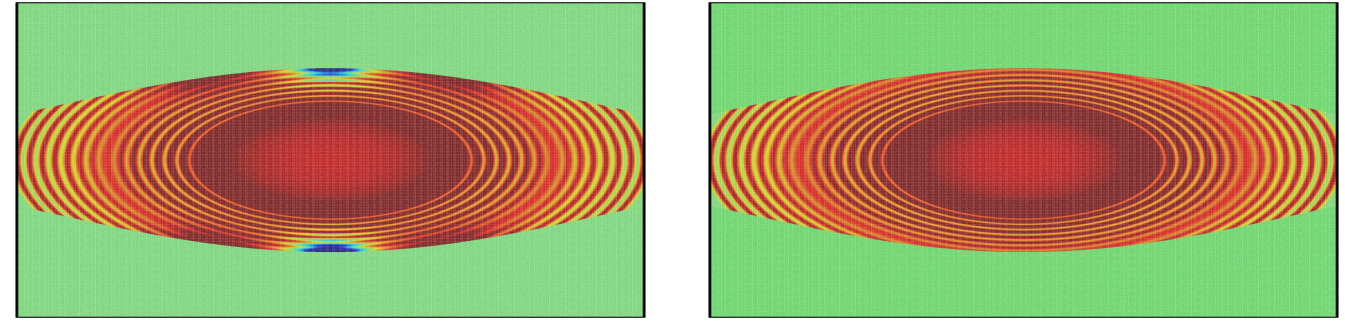

$$
K=600
$$
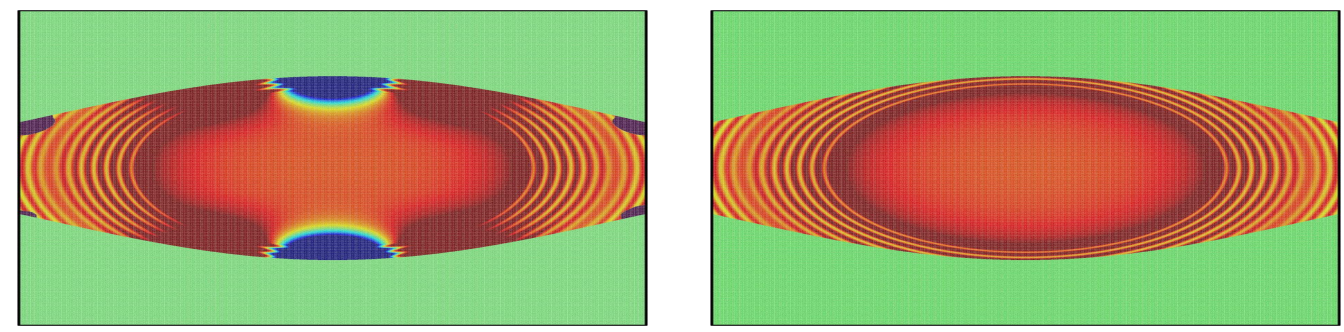

$$
K=760
$$

Figure 4: These are the result of Example 5, building learning boundary condition for a curve boundary. The first column is the numerical solution and the second column corresponds to the precise solution acquired from solving the equation in a larger region.

supported in $r<0.125$ and the dependent set of $w_{n 0}^{k+1}$ is $\left\{w_{n 0}^{k}, w_{n 1}^{k}, w_{n 2}^{k}, w_{n 0}^{k-1}, w_{n 1}^{k-1}, w_{n 2}^{k-1}\right\}$. Once the coefficients $a_{i j}^{l}$ are acquired without the stability criterion (4.16), so is obtained the iterative matrix $C$.

Then solved by power method, the ten largest absolute values of $C^{\prime}$ s eigenvalues are: 


\section{$\begin{array}{lllll}1.0442227 & 1.0301901 & 1.0161737 & 1.0066512 & 1.0066512\end{array}$

$\begin{array}{lllll}0.9997243 & 0.9997243 & 0.9997080 & 0.9997080 & 0.9994696\end{array}$

As we can see, the spectral radius of $C$ in this case is 1.0442227 which is larger than 1 and there are five eigenvalues that are larger that 1 , which implies the differential scheme is not stable.
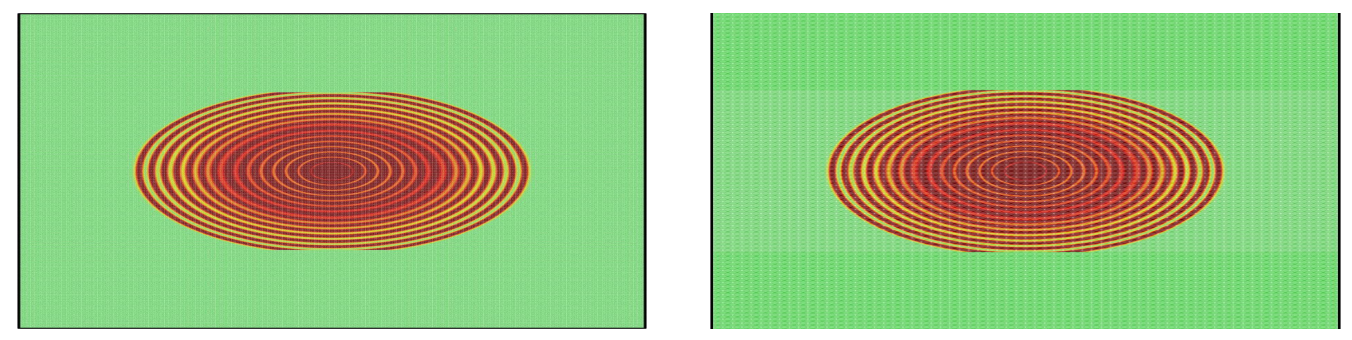

$$
K=280
$$
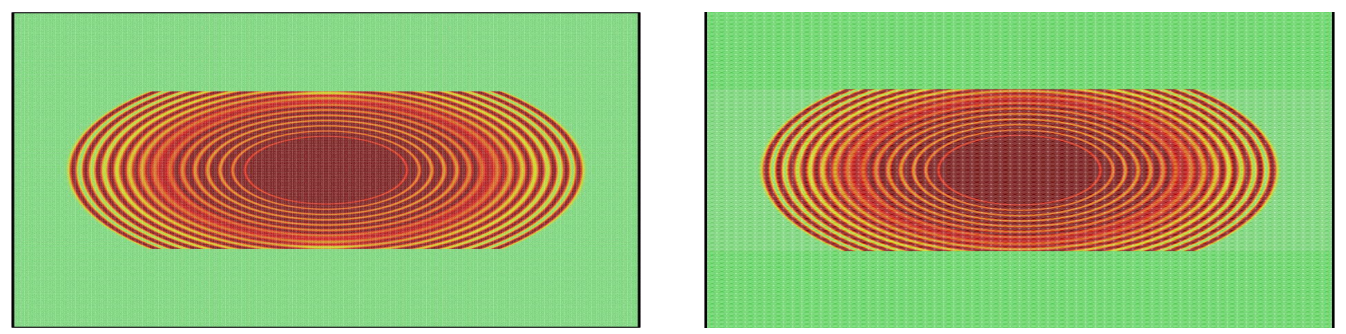

$K=440$
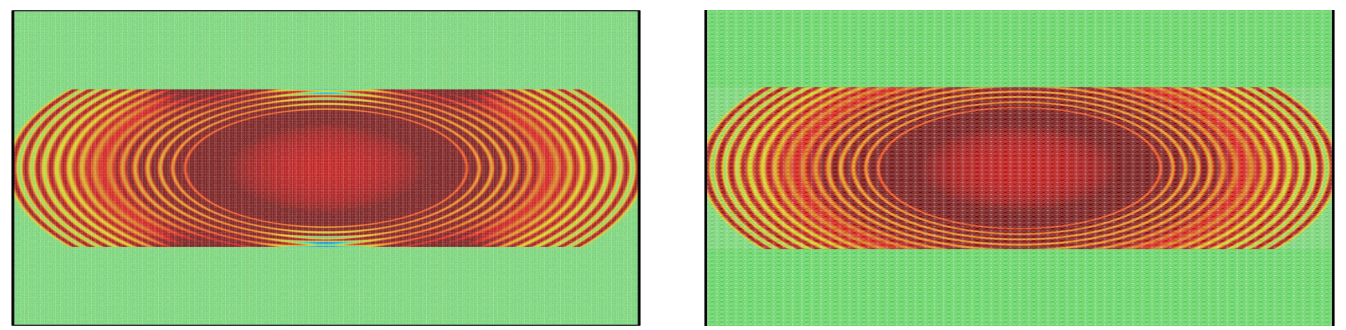

$K=600$
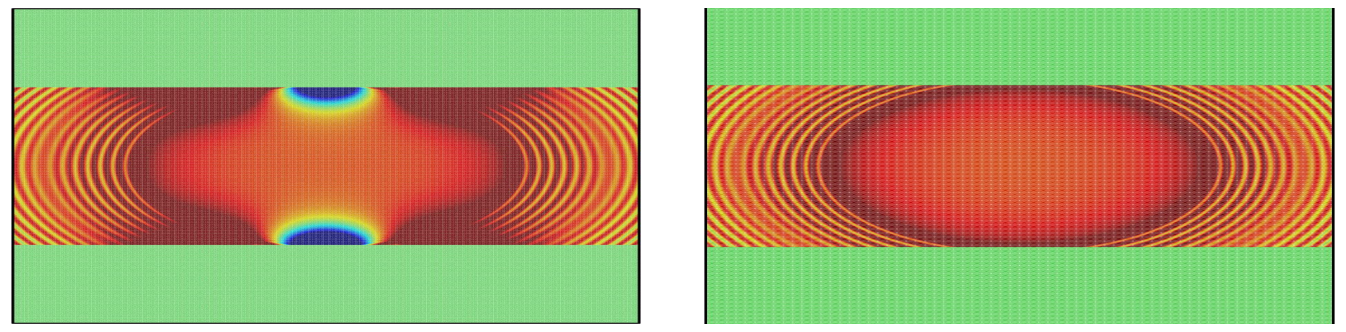

$$
K=760
$$

Figure 5: These are the result of Example 5, building learning boundary condition for the straight boundary. The first column is the numerical solution and the second column corresponds to the precise solution acquired from solving the equation in a larger region. 

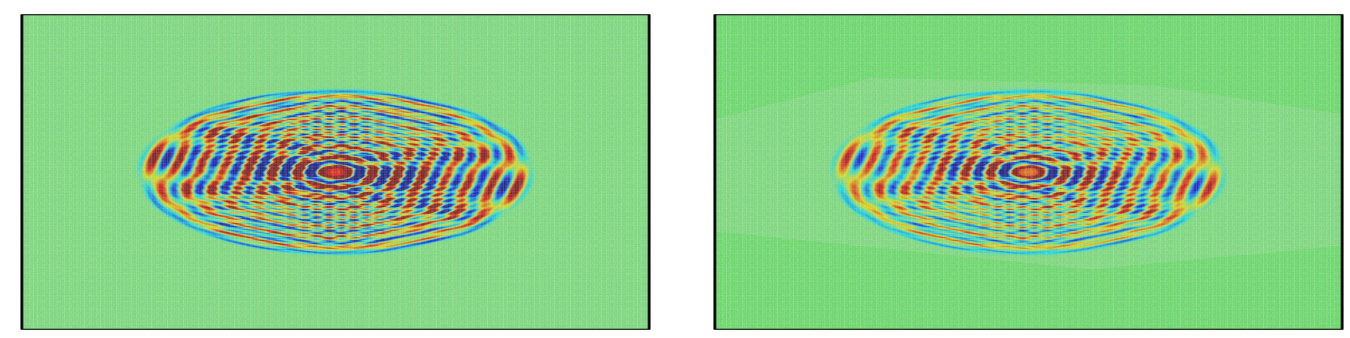

$K=280$
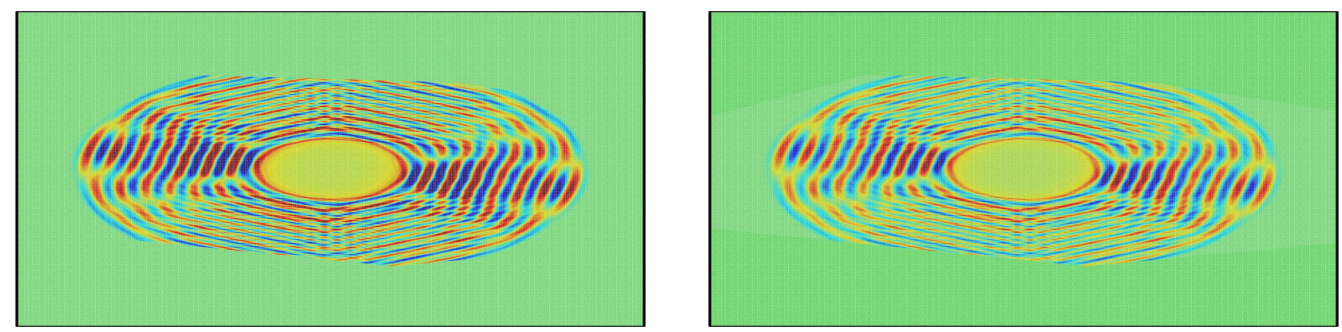

$$
K=440
$$

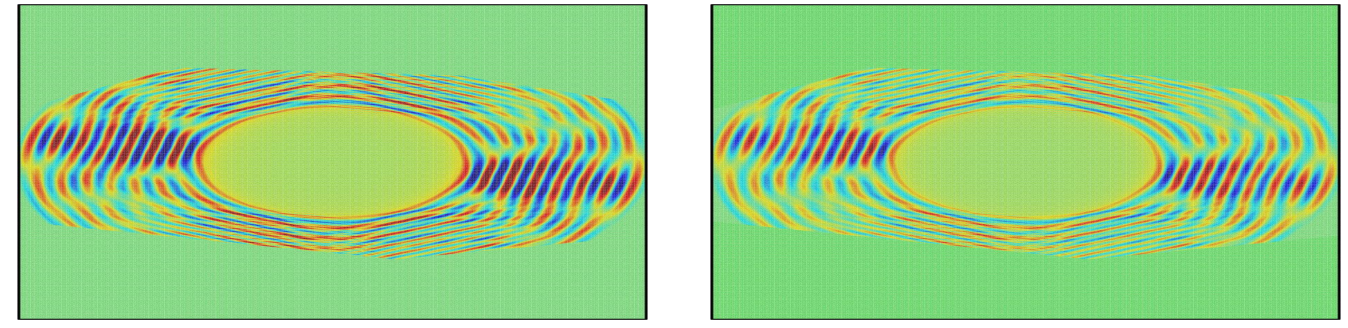

$K=600$

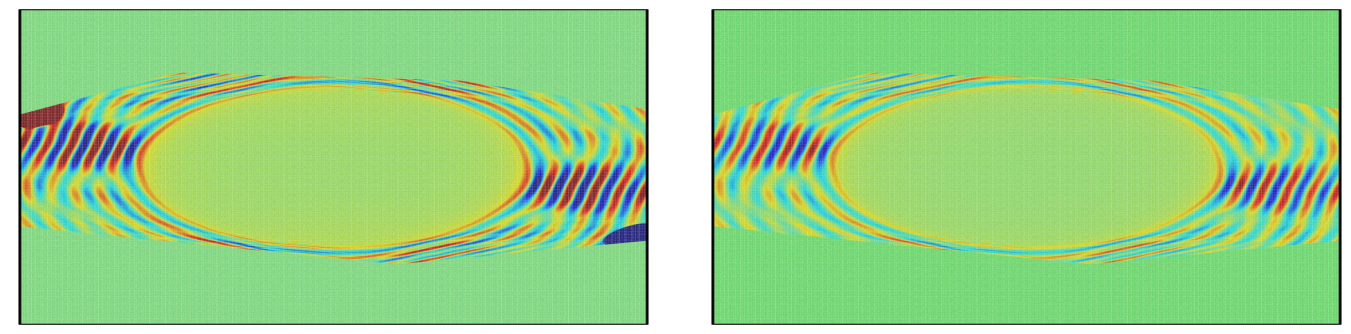

$$
K=760
$$

Figure 6: These are the result of Example 6, building learning boundary condition for the polygonal line boundary. The first column is the numerical solution and the second column corresponds to the precise solution acquired from solving the equation in a larger region.

\subsubsection{Example 8}

We build the same system as in Example 7 (Section 4.4.3) and yield the same learning data set. But this time the regression under the constraint (4.16) is reproduced. Then the 

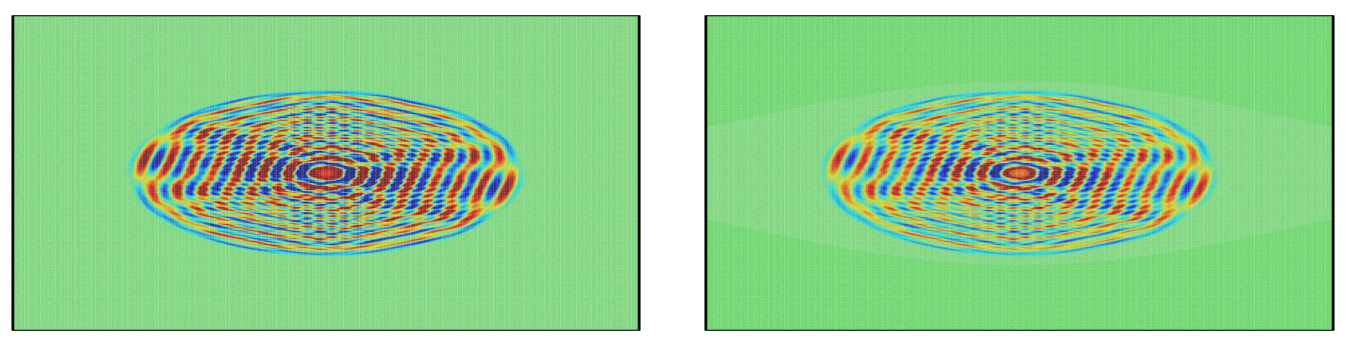

$$
K=280
$$
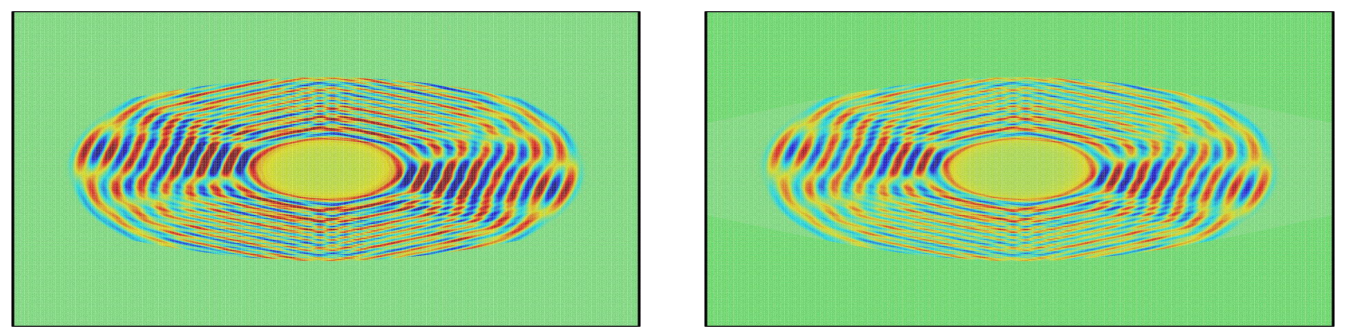

$$
K=440
$$
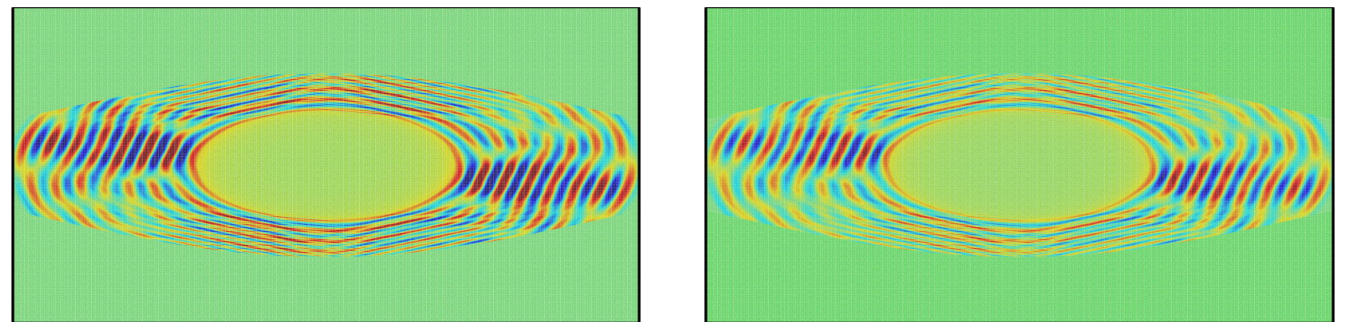

$$
K=600
$$
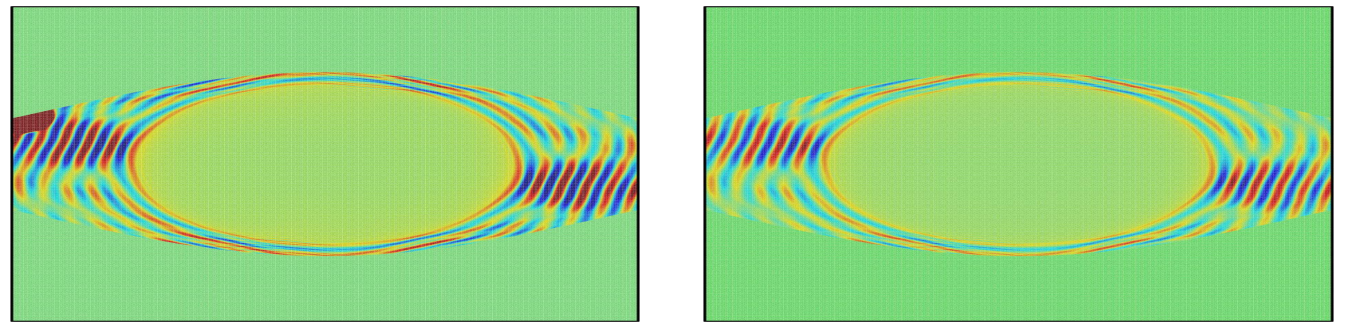

$$
K=760
$$

Figure 7: These are the result of Example 6, building learning boundary condition for the curve boundary. The first column is the numerical solution and the second column corresponds to the precise solution acquired from solving the equation in a larger region.

ten largest absolute values of $C^{\prime}$ s eigenvalues read:

$\begin{array}{lllll}1.0007314 & 0.9998302 & 0.9998302 & 0.9998161 & 0.9998161 \\ 0.9997712 & 0.9997712 & 0.9996096 & 0.9996096 & 0.9995813\end{array}$



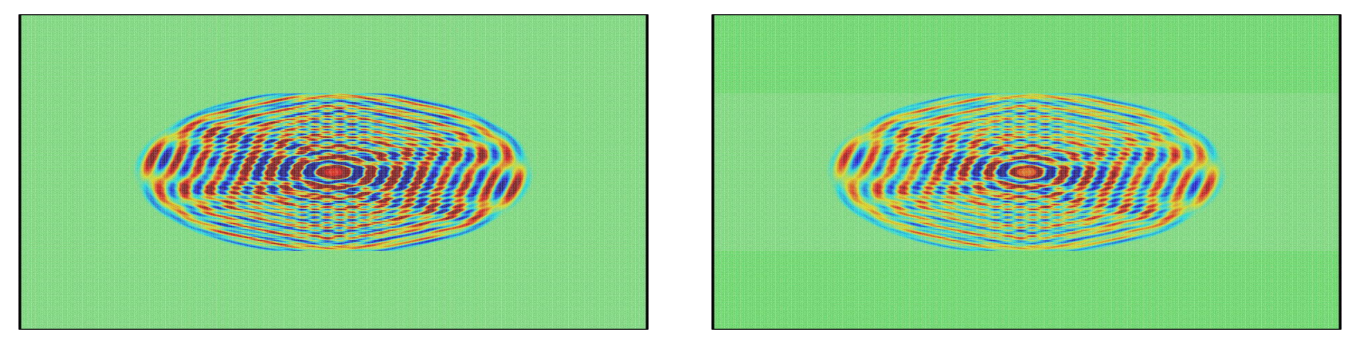

$K=280$
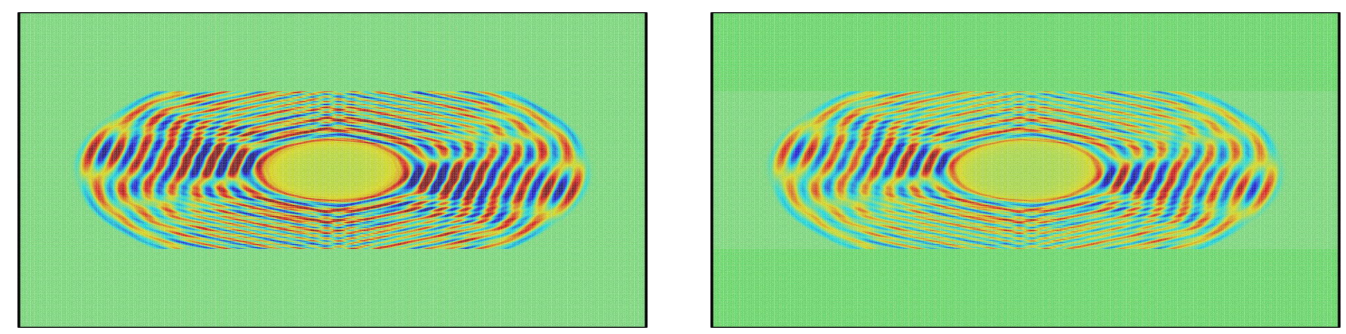

$$
K=440
$$

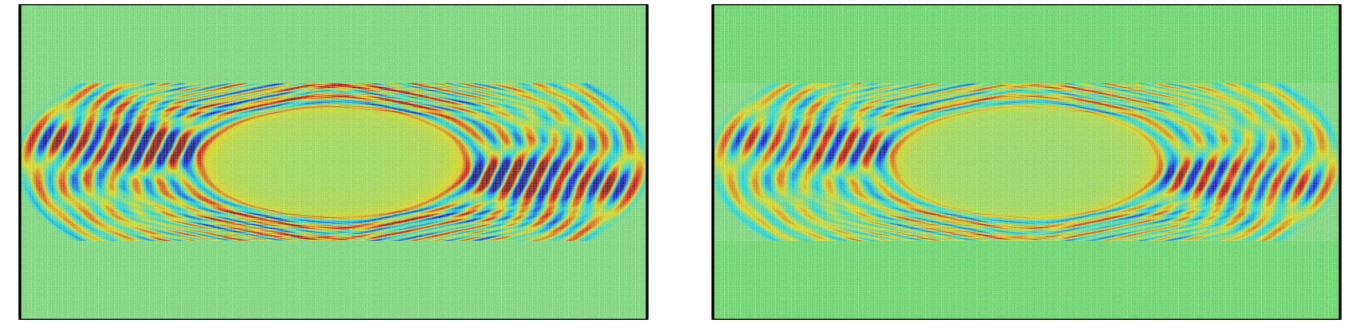

$K=600$

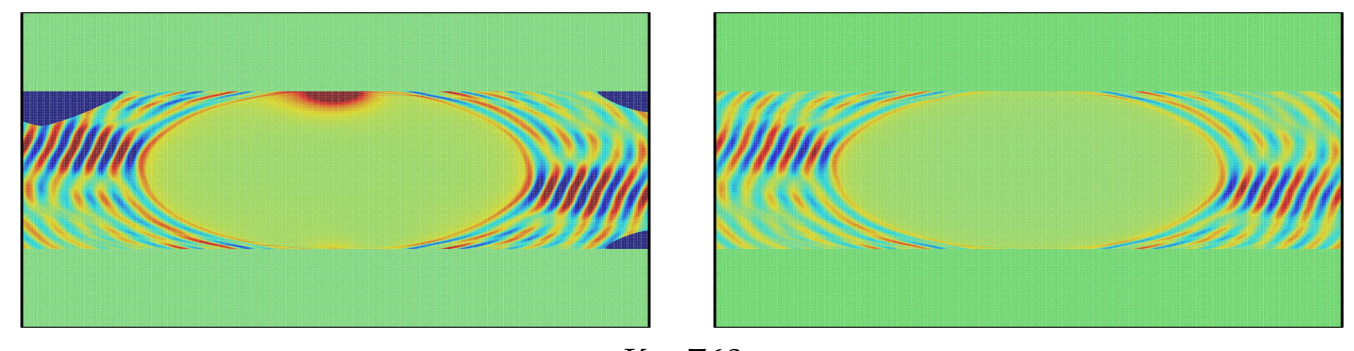

$$
K=760
$$

Figure 8: These are the result of Example 6, building learning boundary condition for the straight boundary. The first column is the numerical solution and the second column corresponds to the precise solution acquired from solving the equation in a larger region.

A comparison diagram is given below in Fig. 9.

As we can see in the Fig. 9, the absolute values of $C^{\prime}$ s eigenvalues have been lowered by performing the regression under the condition (4.16). So we rerun the same test experiments in Example 5 and this time the Lbc performs better. 


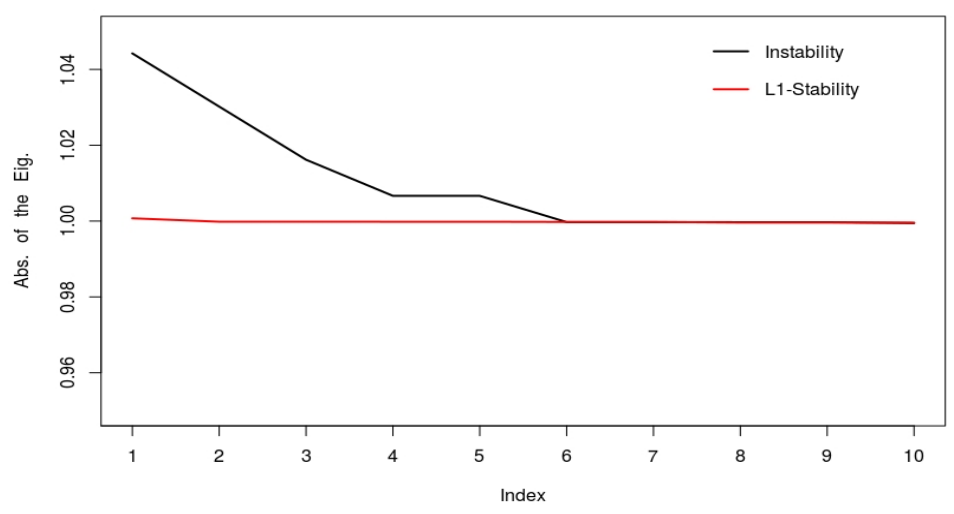

Figure 9: Ten largest eigenvalues of $C$.

\subsubsection{Example 9}

Example 5 in Section 4.4.1 is investigated. With the same discrete form used, we have the same learning data set but the optimization problem is under the constraint (4.16). Even though for a $1200 \times 600$ space grid the calculation of the eigenvalues of the iteration matrix is too costing to be done $\left(\operatorname{dim} C=1.44 \times 10^{6}\right)$, still we can see the stability of the new learning boundary condition and how important the constraint is, see the results of the experiments illustrated in Figs. 10, 11 and 12.

In these figures, the reflected waves are reduced to a very low intensity in all three cases: polygonal line, curve and straight boundary. Recall that the stability condition (4.16) was raised only in case of straight boundary before, however the straightforward replication to the irregular boundary cases also works, at least in these experiments. Therefore a practical method of building learning boundary condition for any region for wave equations is deduced.

\subsection{Remark}

For the boundary condition of the corner, as we can see in those experiments, if simply regarded as an ordinary node of one of its boundary, the corner node leads to some reflection, and however our method reduces this phenomenon. But for a continuous boundary, instead of isolated nodes like the endpoints of the interval or corners of rectangle, the Lbc we build brings about the divergency of the solution without the constraint of stability criterion, especially when the incident wave is generated from the initial value that differs from the learning function. Even for the straight boundary, neither can we get a good result. Once the stability analysis of the previous method is put into force and a constraint of the dependent coefficients $a_{\alpha \beta}^{j}$ is made, it effectively reduces this instability. 

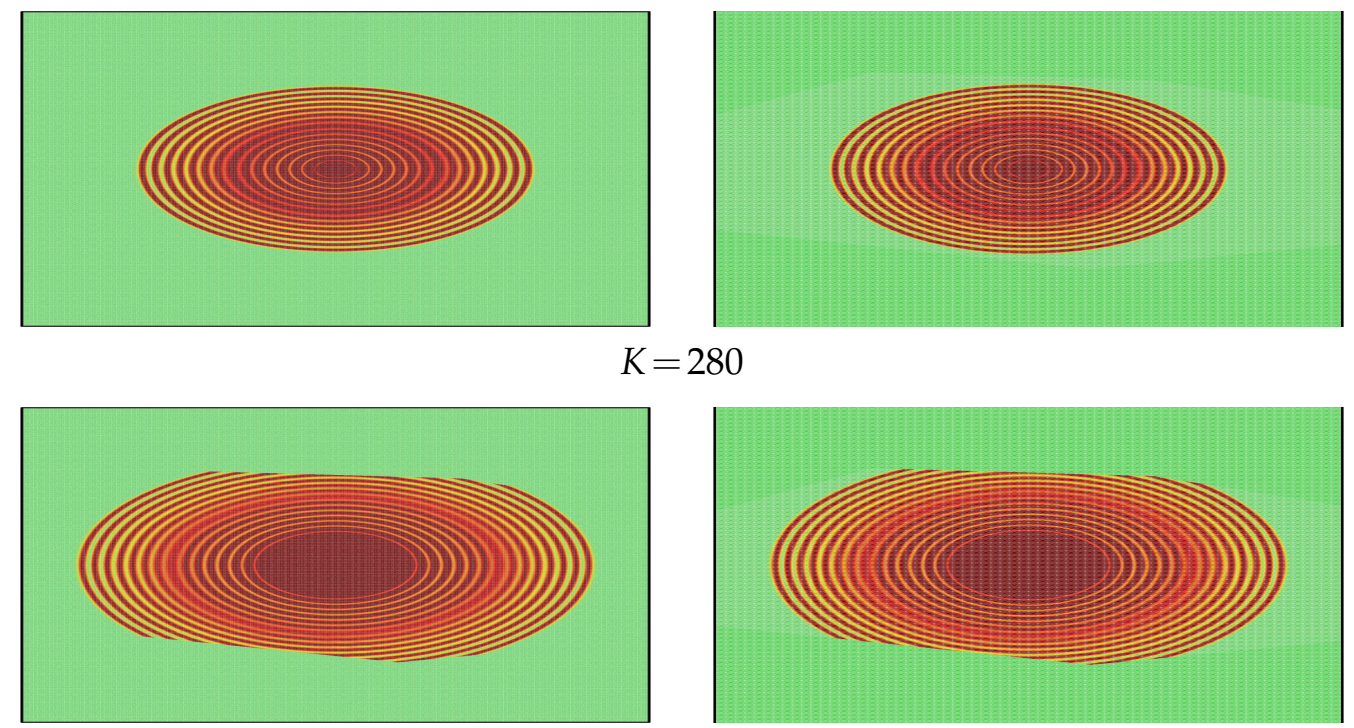

$K=440$
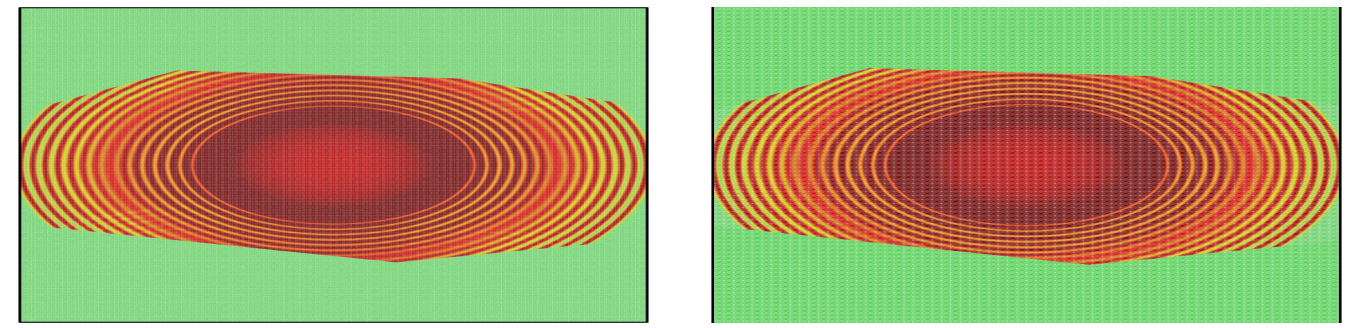

$K=600$
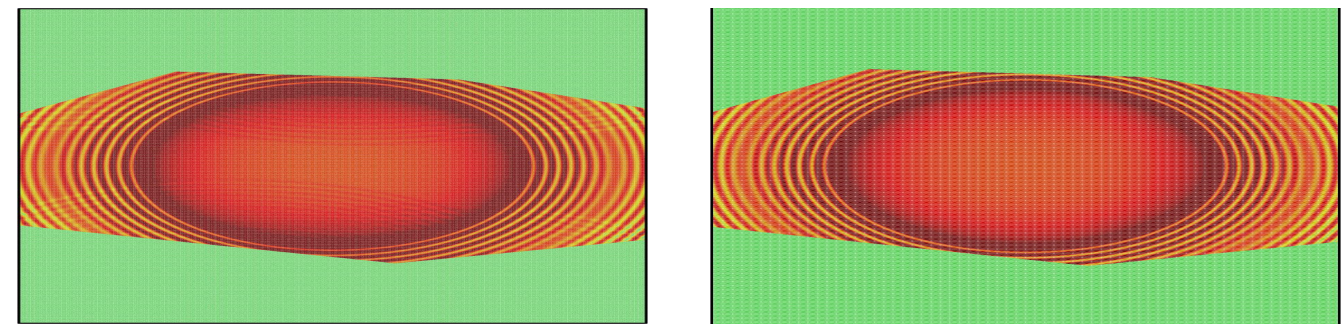

$$
K=760
$$

Figure 10: These are the result of Example 9, building the learning boundary condition coming from (5.13) for the polygonal line boundary. The first column is the numerical solution and the second column corresponds to the precise solution acquired from solving the equation in a larger region.

\section{Generalization ability and ridge regression}

In last section, the stability criterion is introduced, with which the learning boundary condition have long-time stability. However, in this section the problem raised in Section 

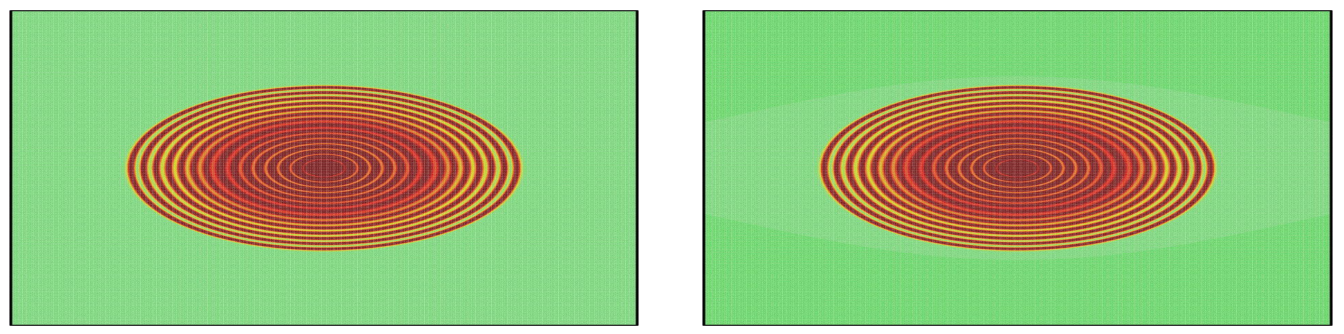

$K=280$
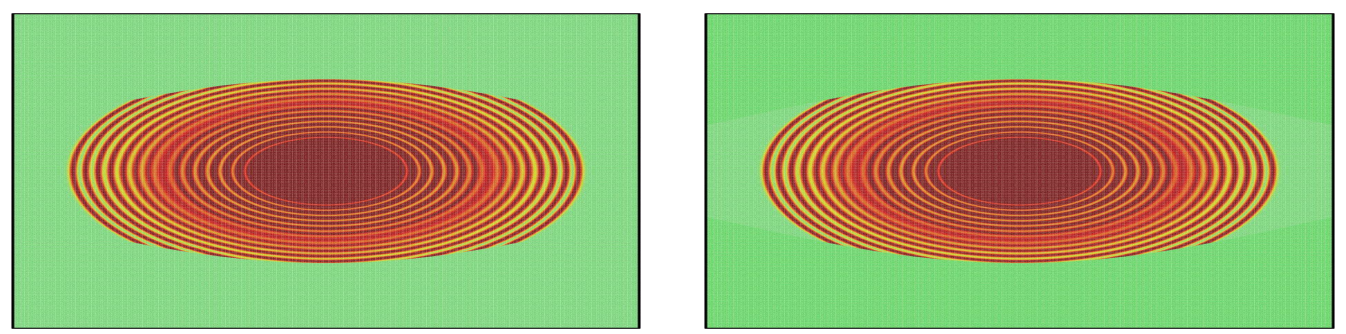

$K=440$

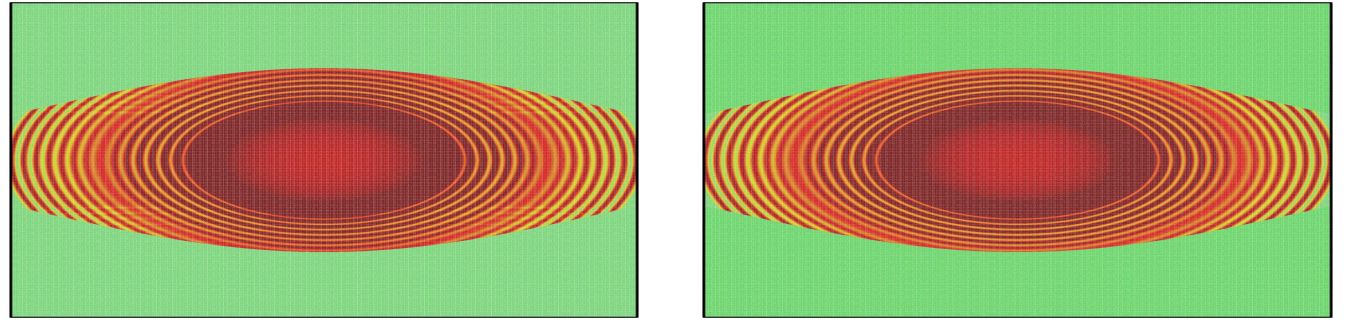

$K=600$
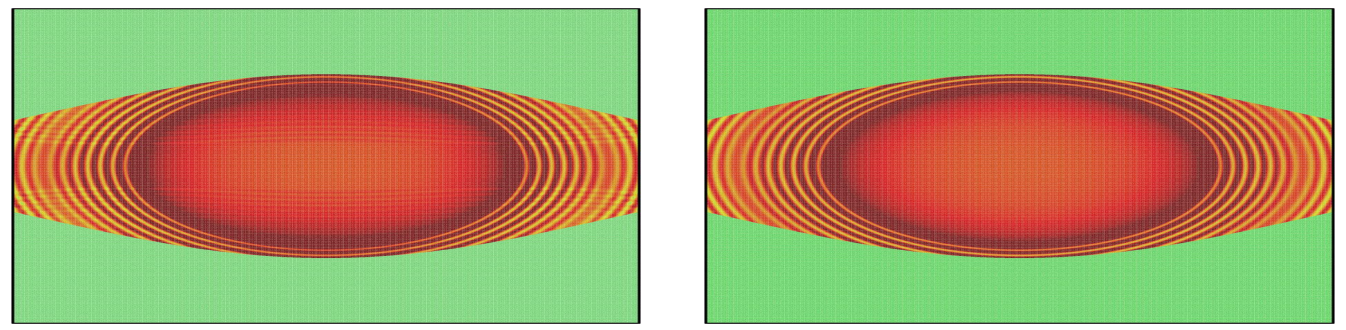

$$
K=760
$$

Figure 11: These are the result of Example 9, building the learning boundary condition coming from (5.13) for the curve boundary. The first column is the numerical solution and the second column corresponds to the precise solution acquired from solving the equation in a larger region.

4.2 is viewed from another direction. First we point out that the stability criterion has only been discussed when dealing with straight boundary and there is no idea whether it still works with irregular boundary, and the export of the criterion is nothing easier than the perfectly absorbing boundary condition. More or less it contradicts our wish of 

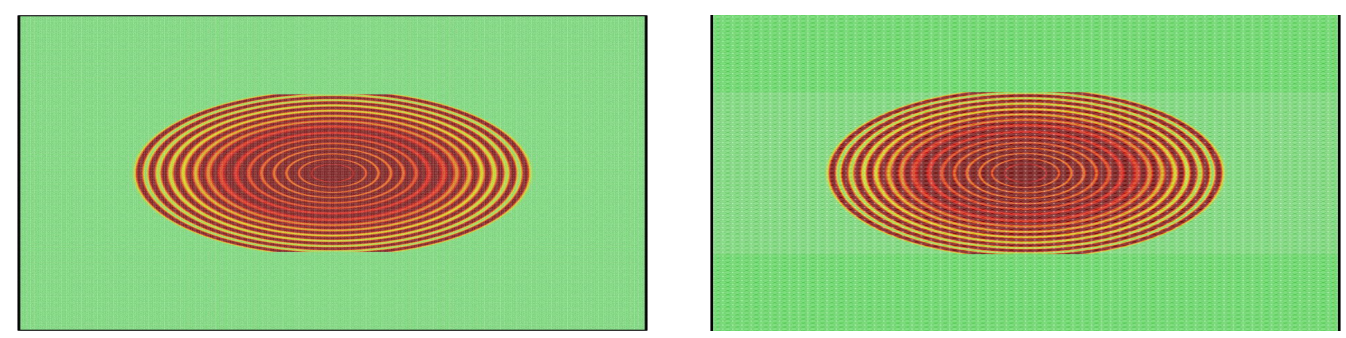

$K=280$
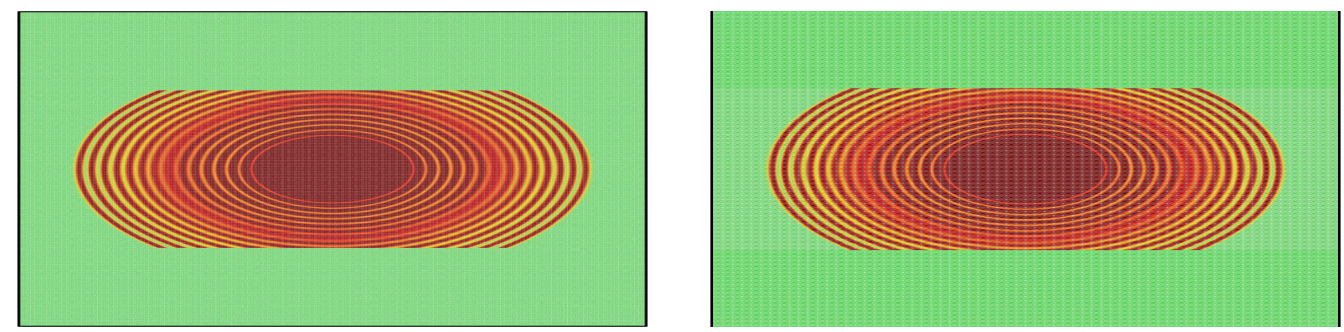

$$
K=440
$$

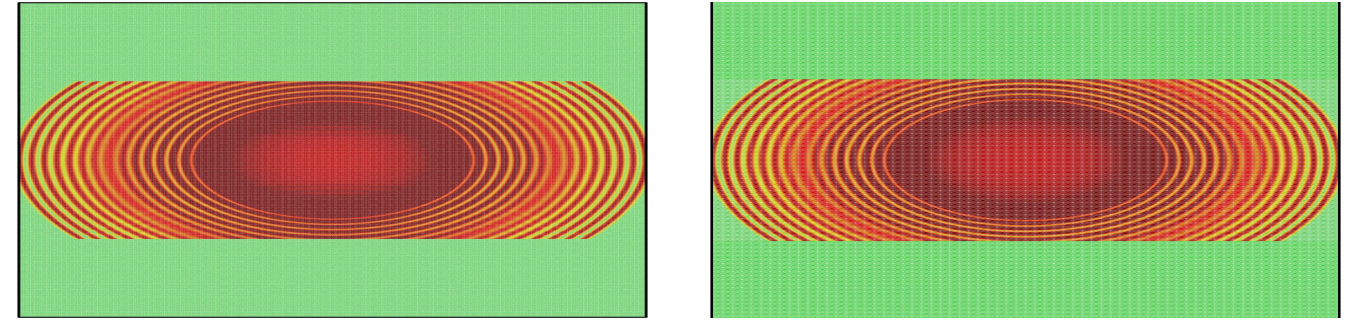

$$
K=600
$$
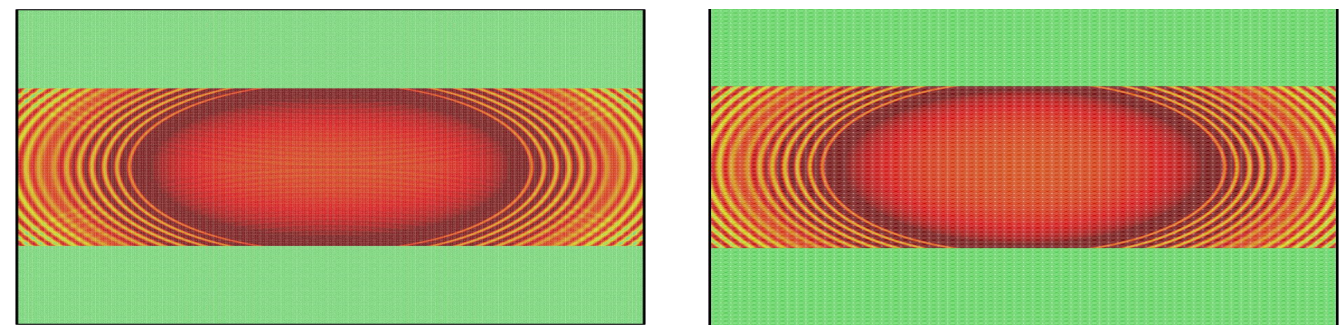

$$
K=760
$$

Figure 12: These are the result of Example 9, building the learning boundary condition coming from (5.13) for the straight boundary. The first column is the numerical solution and the second column corresponds to the precise solution acquired from solving the equation in a larger region.

not analytical-costing. So in this section we take the pathosis of the data set as the major cause of the problem, and settle with Ridge Regression method. 


\subsection{Ridge regression}

Carefully compare Figs. 6, 7, 8 with Figs. 3, 4, 5, one can find that the solution whose initial-values match the learning functions is better than the others. We may infer that since our learning set comes from just one solution, the learning boundary condition don't properly predict the behaviour of those we haven't learned from, which means a poor generalization ability.

In fact, as we can only minimize the error of those finite solution sampled, the coefficients acquired by the empirical risk (2.12) dose not satisfy the minimum value of the population risk (2.11). The error between the empirical risk (2.12) and the population risk (2.11) is called the generalization error, which is the main difficulty preventing the application of the learned model on unlearned data. Usually the generalized error can be divided into two part. The first one is the error between the distribution from which the data is generated and the one implied by the model. In LBCs, since the data is generated artificially by the numerical solution of the equation, this error can be omitted. The other part is the truncation error, if we let

$$
\mathbb{D}\left(b_{i j}^{l}, w_{0 \beta}^{k+1}\right)=\left|w_{0 \beta}^{k+1}-\sum_{\mathscr{D}_{0 \beta}^{k+1}} b_{i j}^{l} w_{i j}^{k+1-l}\right|^{2}
$$

with respect to a sampling obeying $\rho$, Monte Carlo analysis tells us that the difference is:

$$
\begin{aligned}
\mathbb{E}\left(\text { Loss }_{p o p}-\operatorname{Loss}_{\text {emp }}\right)^{2} & =\mathbb{E}\left(\int \mathbb{D}\left(b_{i j}^{l}, w_{0 \beta}^{k+1}\right) \rho\left(d w_{0 \beta}^{k}\right)-\frac{1}{N} \sum_{m, k} \mathbb{D}\left(b_{i j, m}^{l} w_{0 \beta}^{k+1}\right)\right)^{2} \\
& =\frac{1}{N}\left[\int_{\rho} \mathbb{D}^{2}\left(b_{i j}^{l}, w_{0 \beta}^{k+1}\right)-\left(\int_{\rho} \mathbb{D}\left(b_{i j}^{l}, w_{0 \beta}^{k+1}\right)\right)^{2}\right] \\
& =\frac{1}{N} \operatorname{Var}\left(\mathbb{D}\left(b_{i j}^{l}, w_{0 \beta}^{k+1}\right)\right),
\end{aligned}
$$

which means that to reduce the generalization error between the population risk and the empirical risk is to reduce the variance of the model. Therefore, some bias may be traded for the variance of the model. Thus a Tikhonov regularization is applied:

$$
\text { Tikhonov: } a_{i j}^{l} \in \min _{\left\|a_{\alpha \beta}^{j}\right\|_{2}<r} \operatorname{Loss}_{e m p}\left(b_{i j}^{l}\right) \text {. }
$$

Invented independently in many different contexts, ridge regression, also called Tikhonov regularization, was first expounded by E. Hoerl in [8], and following whom it is known in the statistical literature as ridge regression $[20,21]$. The choice of coefficient $r$ is always a tough question but however, in our paper, we simply take $\|b\|_{2}<1$ as the constraint for an obvious physical reason. In Section 2 it has been shown how to solve this problem. 


\subsection{Numerical experiments}

\subsubsection{Example 10}

We build the same system as in Example 7 (section 4.4.3) and this time we produce the ridge regression on the data set instead. Observe that this condition is weaker than (4.16): $\|x\|_{1}<1 \Longrightarrow\|x\|_{2}<1$. Both the condition numbers of the regression matrix and the eigenvalues of the iteration matrix are computed and illustrated below. As shown in Fig. 13, those regression matrixs have large condition numbers, which confirms our assumption of large variance. But for ridge regression, the condition number of the regressive matrix is much smaller. At least, no matter the method works or not, the ridge regression markedly changes the numerical illness of the problem.

Also the ten largest eigenvalues of the iteration matrix $C$ are evaluated as we did in Section 4.3 in Fig. 14.

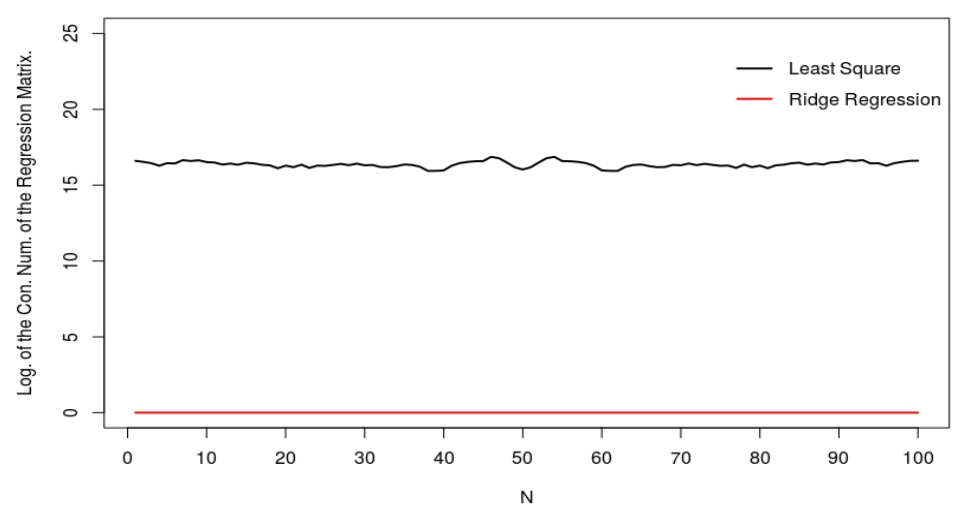

Figure 13: The Logarithms of Condition Numbers of regressive matrix.

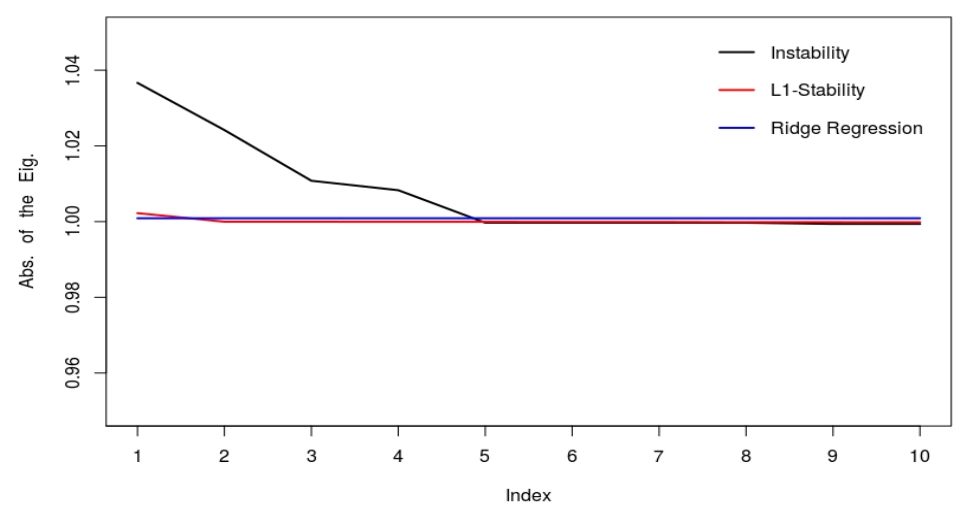

Figure 14: Ten largest eigenvalues of iterative matrix. 

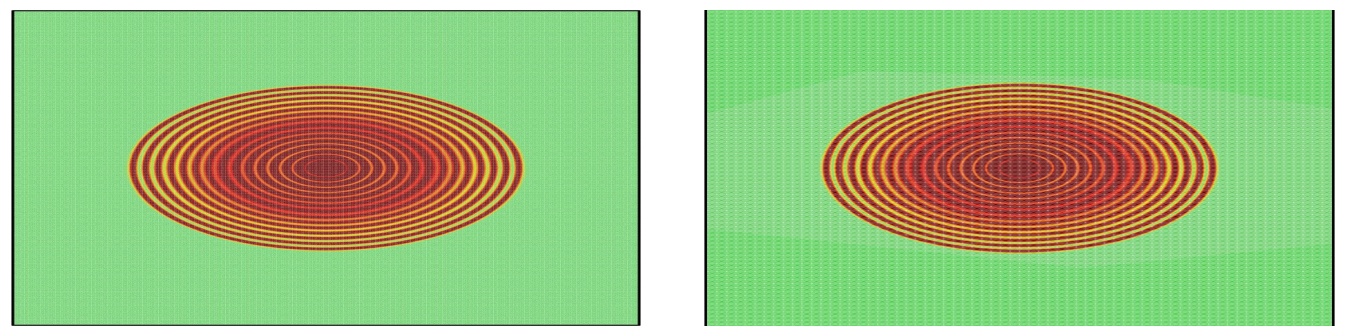

$$
K=280
$$
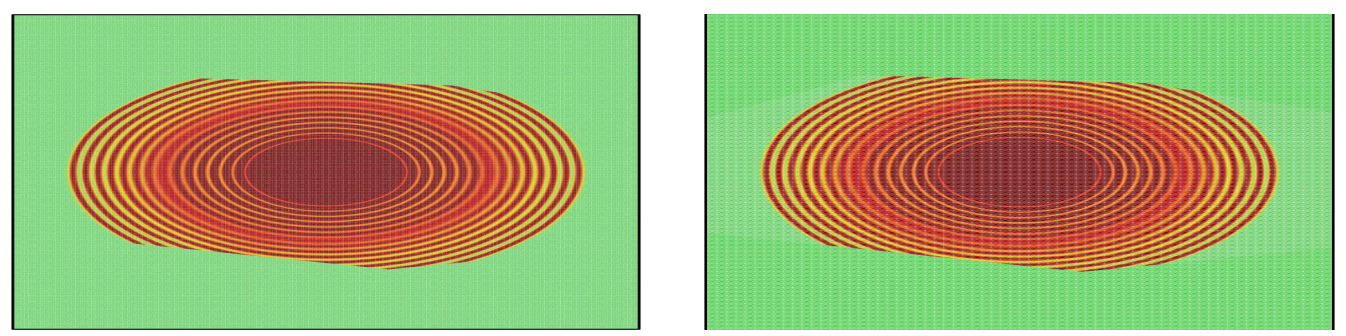

$$
K=440
$$
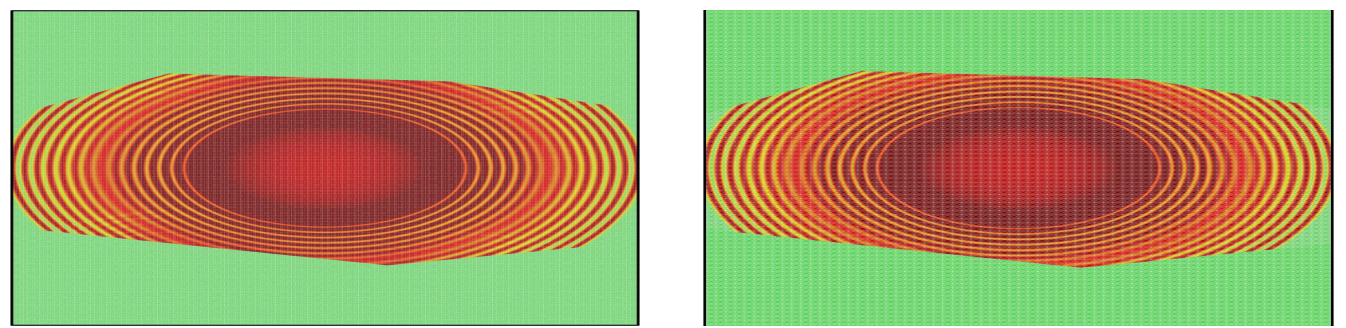

$$
K=600
$$
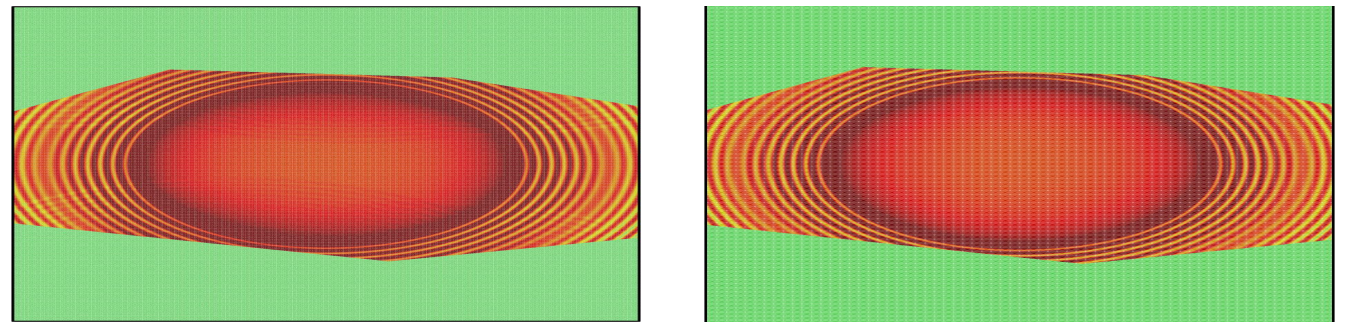

$$
K=760
$$

Figure 15: These are the result of Example 11, building the learning boundary condition coming from (5.2) for the polygonal line boundary. The first column is the numerical solution and the second column corresponds to the precise solution acquired from solving the equation in a larger region.

\subsubsection{Example 11}

As Example 9 (Section 4.4.5), Example 5 is redone with the ridge regression method. The results of the numerical solution are shown in Figs. 15, 16 and 17. 

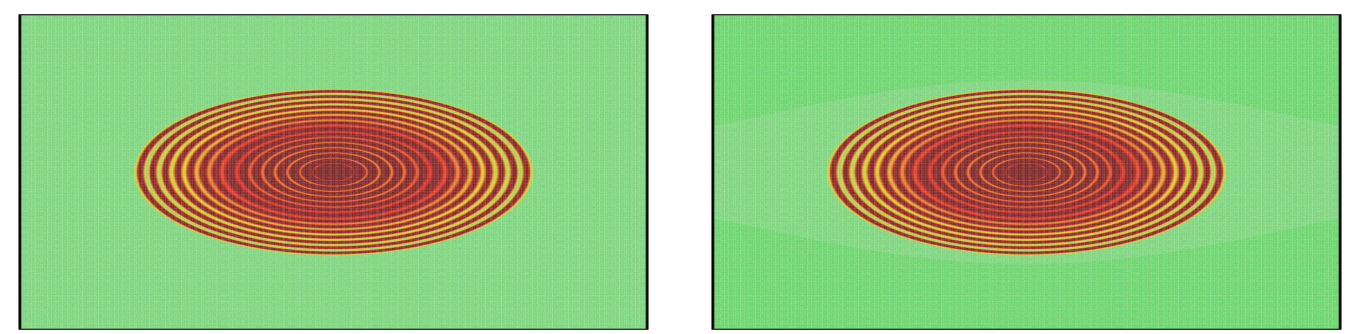

$K=280$
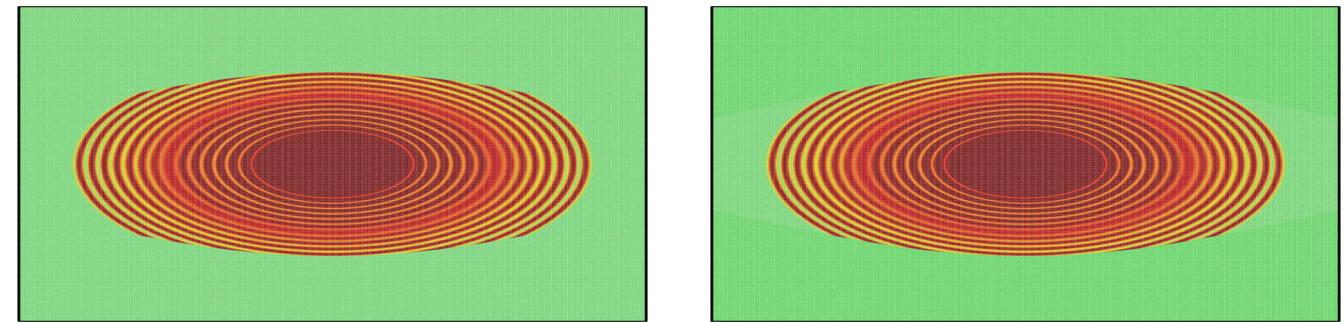

$K=440$
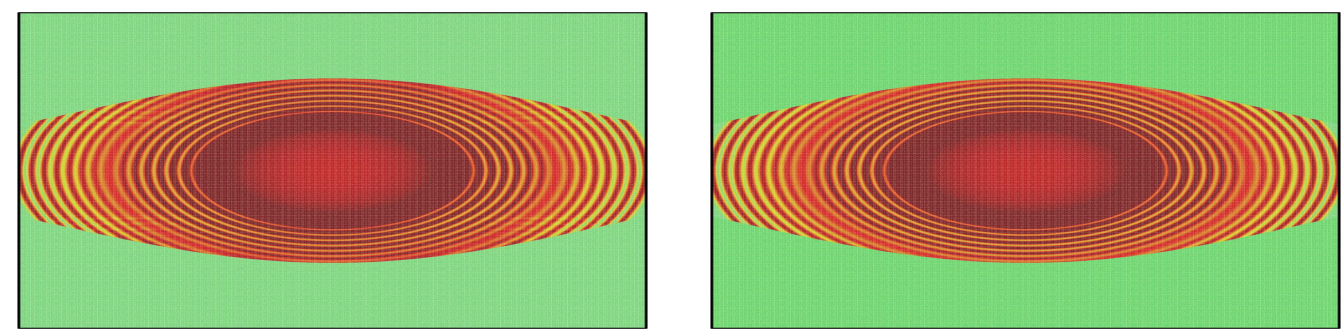

$K=600$
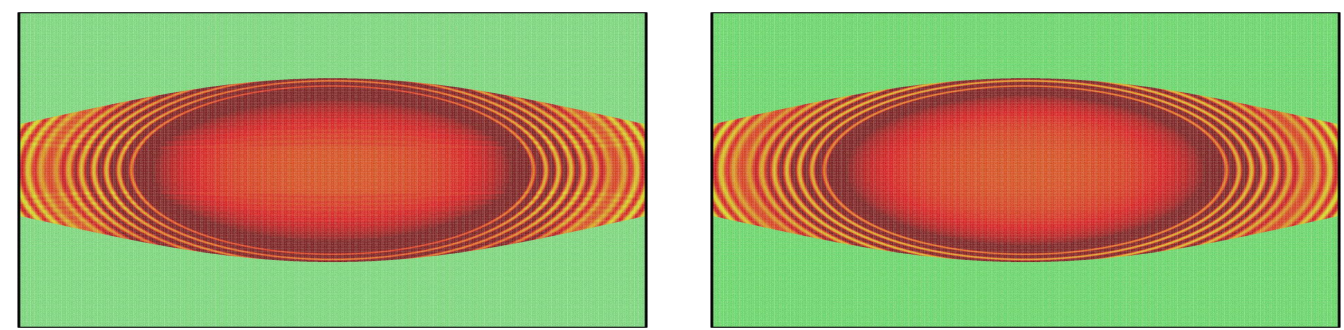

$$
K=760
$$

Figure 16: These are the result of Example 11, building the learning boundary condition coming from (5.2) for the curve boundary. The first column is the numerical solution and the second column corresponds to the precise solution acquired from solving the equation in a larger region.

As illustrated in these figures. These learning boundary condition efficaciously reduces the reflex wave also. Now another method for building learning boundary condition is inferred by the regression ridge method. 

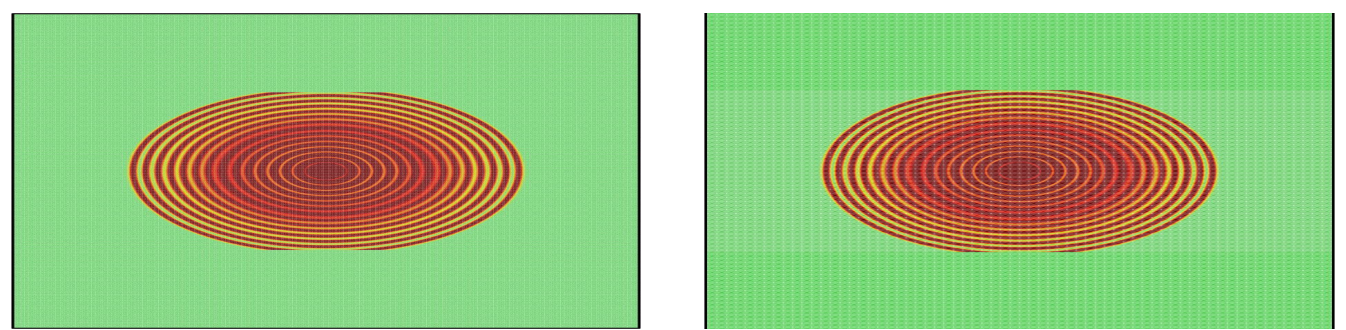

$$
K=280
$$

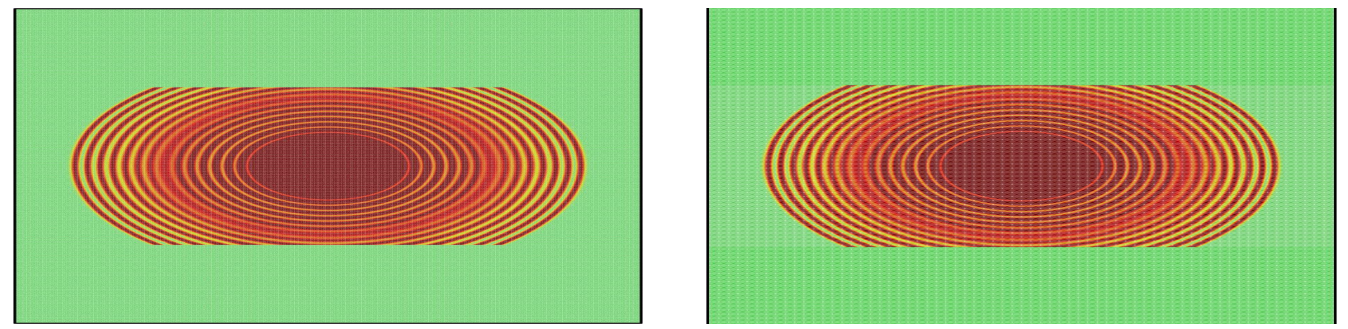

$$
K=440
$$
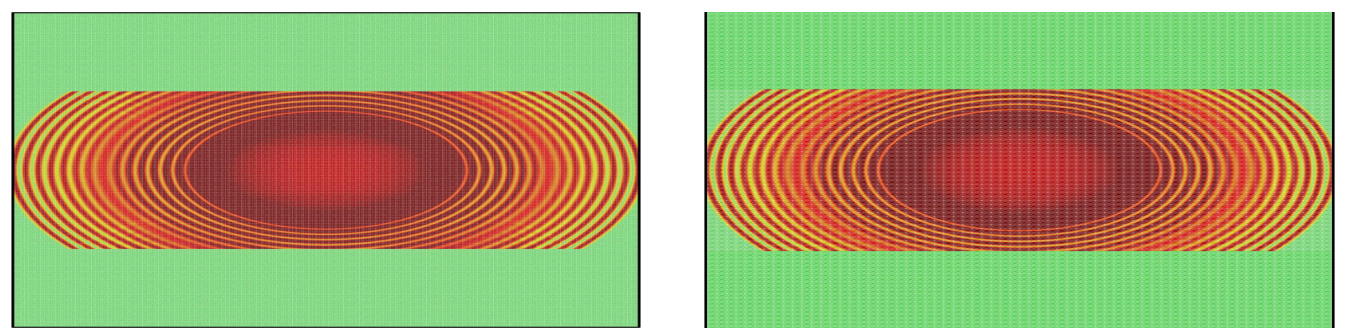

$$
K=600
$$
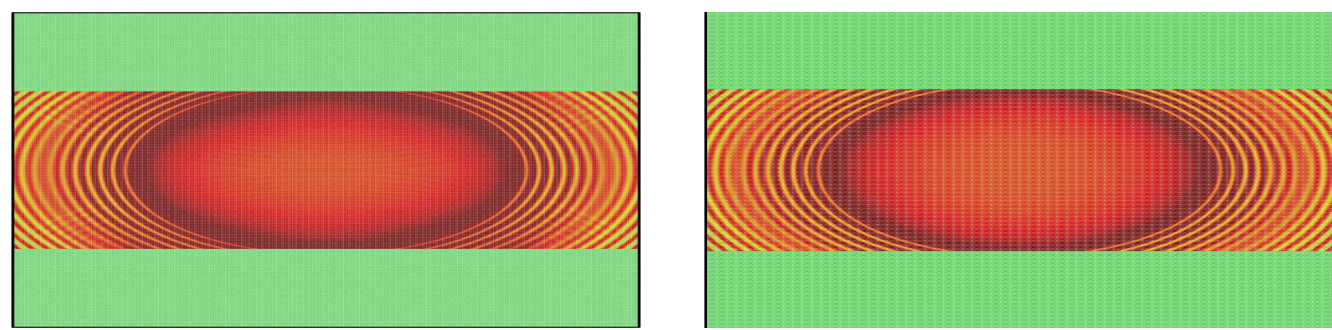

$$
K=760
$$

Figure 17: These are the result of Example 11, building the learning boundary condition coming from (5.2) for the straight boundary. The first column is the numerical solution and the second column corresponds to the precise solution acquired from solving the equation in a larger region.

\subsection{Remark}

Fig. 14 shows that both the stability criterion and ridge regression, at least in this case, help us gain the stability. The only difference between the stability criterion and ridge 
regression is the motivation. Latter one comes from machine learning and its adhibition in more complicating case, like other kinds of partial differential equations, is realizable.

\section{Conclusions}

In this paper, a new machine learning method to build learning boundary condition for wave equations is proposed. Compared with former methods, our machine learning method has three advantages. First, instead of the costing theoretical analysis, regression is a much briefer process, and its further generalization like building learning boundary condition for Maxwell function or elastic wave equations is realizable. Even if we don't have the stability criterion like the one given in Section 4.3, carefully adding one regularization term in regression according to the property of the data set will work well too. Second, not always the boundary that we face are regular. And our method can effectively deal with all various boundary in any dimensions. Finally, when we have some information about the incidence beforehand, that is the $\rho$ in (2.11), we can choose the learning function obeying this distribution and reach a targeted learning boundary condition. It will enhance the absorbing ability and do better than an average $A B C$.

\section{Acknowledgements}

This work is supported by the National Natural Science Foundation of China (Grant Nos. 11671312, 91630313) and by the Natural Science Foundation of Hubei Province No. 2019CFA007. The numerical calculations in this paper have been done on the supercomputing system in the Supercomputing Center of Wuhan University.

\section{References}

[1] Alessandrini Giovanni, De Hoop MaArten V., Faucher Florian, Gaburro ROMINA AND SINCICH EVA, Inverse problem for the helmholtz equation with cauchy data: reconstruction with conditional well-posedness driven iterative regularization, ESAIM: M2AN, 53(3) (2019), pp. 1005-1030.

[2] ZDENĚK P. BAŽANT, Spurious reflection of elastic waves in nonuniform finite element grids, Comput. Methods Appl. Mech. Eng., 16(1) (1978), pp. 91-100.

[3] AntOINE BENOIT, Semi-group stability of finite difference schemes in corner domains, Numer. Math. Theory Methods Appl., 11(3) (2018), pp. 618-654.

[4] JEAN-PIERRE BERENGER, A perfectly matched layer for the absorption of electromagnetic waves, J. Comput. Phys., 114(2) (1994), pp. 185-200.

[5] B. GustafsSON, H.-O.KREISS, AND A. SundSTRM, Stability theory of difference approximations for mixed initial boundary value problems II, Math. Comput., 26(119) (1972).

[6] ZEKAI CELEP AND ZDENĚK P. BAŽANT, Spurious reflection of elastic waves due to gradually changing finite element size, Int. J. Numer. Methods Eng., 19(5) (1983), pp. 631-646. 
[7] Albert Chenn, A reflectionless discrete perfectly matched layer, J. Comput. Phys., 381 (2019), pp. 91-109.

[8] E. HOERL AND W. KeNNARD, Ridge regression: Biased estimation for nonorthogonal problems, Technometric, 12 (1970).

[9] BJÖRN ENGQUIST AND ANDREW MAJDA, Absorbing boundary conditions for numerical simulation of waves, Proceedings of the National Academy of Sciences, 74(5) (1977), pp. 1765-1766.

[10] DAN GIVOli AND DAN COHEN, Nonreflecting boundary conditions based on kirchhoff-type formulae, J. Comput. Phys., 117(1) (1995), pp. 102-113.

[11] Boris GuREVICH, N. DAI, A. VAFIDIS, AND E. R. KANASEWICH, Wave Propagation in heterogeneous, porous media: A velocity-stress, finite difference method; discussion and reply, Geophysics, 61(4) (1996), pp. 1230-1232.

[12] Thomas Hagstrom, Dan Givoli, Daniel Rabinovich, and Jacobo BielaK, The double absorbing boundary method, J. Comput. Phys., 259 (2014), pp. 220-241.

[13] LAURENCE HALPERN, Absorbing boundary conditions for the discretization schemes of the onedimensional wave equation, Math. Comput., 38(158) (1982).

[14] R. HIGDON, Radiation boundary conditions for elastic wave propagation, SIAM J. Numer. Anal., 27(4) (1990), pp. 831-869.

[15] ROBERT L. HIGDON, Absorbing boundary conditions for difference approximations to the multidimensional wave equation, Math. Comput., 47(176) (1986).

[16] ROBERT L. HigdON, Numerical absorbing boundary conditions for the wave equation, Math. Comput., 49(179) (1987).

[17] M. Defrise, I. DAubechies And C. DeMol, An iterative thresholding algorithm for linear inverse problems with a sparsity constraint, Commun. Pure Appl. Math., 57 (2004).

[18] Lijie Ji, YANLAi Chen AND Zhenli XU, A reduced basis method for the nonlinear poissonboltzmann equation, Adv. Appl. Math. Mech., 11(5) (2019), pp. 1200-1218.

[19] JIEQUAN LI, Fundamentals of lax-wendroff type approach to hyperbolic problems with discontinuities, Adv. Appl. Math. Mech., 11(3) (2019), pp. 571-582.

[20] C. L. MALlOWs, Some comments on cp, Technometrics, 15(4) (1973), pp. 661-675.

[21] DonAlD W. MARQUARDT, Generalized inverses, ridge regression, biased linear estimation, and nonlinear estimation, Technometrics, 12(3) (1970), pp. 591-612.

[22] DANiel Michelson, Stability theory of difference approximations for multidi-mensional initialboundary value problems, Math. Comput., 40(161) (1983).

[23] G. MUR, Absorbing boundary conditions for the finite-difference approximation of the time-domain electromagnetic-field equations, IEEE Transactions on Electromagnetic Compatibility, EMC23(4) (1981), pp. 377-382.

[24] Toby Potter, Jeffrey Shragge, AND David Lumley, Performance and stability of the double absorbing boundary method for acoustic wave propagation, Geophysics, 84 (2018), pp. 1-53.

[25] R. A. RenAUt AND J. Petersen, Stability of wide-angle absorbing boundary conditions for the wave equation, Geophysics, 54(9) (1989).

[26] R. A RENAUT, Absorbing boundary conditions, difference operators, and stability, J. Comput. Phys., 102(2) (1992), pp. 236-251.

[27] R. NOWAK AND M. FIgUeIREDO, Fast wavelet-based image deconvolution using the em algorithm, The Asilomar Conference on Signals, Systems, and Computers, 1 (2001).

[28] R. NOWAK AND M. FIGUEIREDO, An em algorithm for wavelet-based image restoration, Image Processing IEEE Transactions on Image Processing, 12 (2003).

[29] R. NOWAK AND M. FIGUEIREDO, A bound optimization approach to wavelet-based image deconvolution, IEEE International Conference, Septemper 2005. 
[30] JIE SHEN AND Li-LiAn WANG, Some recent advances on spectral methods for unbounded domains, Commun. Comput. Phys., 5(2-4) (2009), pp. 195-241.

[31] Arman Shojaei, Farshid Mossaiby, Mirco Zaccariotto, and Ugo Galvanetto, A local collocation method to construct dirichlet-type absorbing boundary conditions for transient scalar wave propagation problems, Comput. Methods Appl. Mech. Eng., 08 (2019).

[32] ROBERT TIBSHIRANI, Regression shrinkage and selection via the lasso: a retrospective, Journal of the Royal Statistical Society: Series B, 73 (2011).

[33] LLOYD N. TREFETHEN, Instability of difference models for hyperbolic initial boundary value problems, Commun. Pure Appl. Math., 37(3) (1984), pp. 329-367.

[34] LlOYD N. TREFETHEN, Stability of finite-difference models containing two boundaries or interfaces, Math. Comput., 45(172) (1985), pp. 279-300.

[35] ROBERT VICHNEVETSKY, Wave propagation analysis of difference schemes for hyperbolic equations: A review, Int. J. Numer. Methods Fluids, 7(5) (1987), pp. 409-452.

[36] Zhencong ZhaO, Jingyi CHen, XIAOBo LiU, AND BAORUi CHEN, Frequency-domain elastic wavefield simulation with hybrid absorbing boundary conditions, J. Geophys. Eng., 16 (2019), pp. 690-706. 